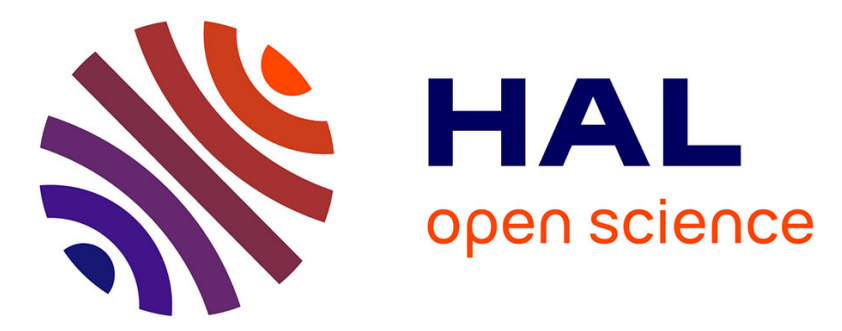

\title{
A variational formulation of constitutive models and updates in non-linear finite viscoelasticity
}

\author{
Eduardo Fancello, Jean-Philippe Ponthot, Laurent Stainier
}

\section{To cite this version:}

Eduardo Fancello, Jean-Philippe Ponthot, Laurent Stainier. A variational formulation of constitutive models and updates in non-linear finite viscoelasticity. International Journal for Numerical Methods in Engineering, 2006, 65 (11), pp.1831-1864. 10.1002/nme.1525 . hal-01004966

\section{HAL Id: hal-01004966 https://hal.science/hal-01004966}

Submitted on 15 Nov 2021

HAL is a multi-disciplinary open access archive for the deposit and dissemination of scientific research documents, whether they are published or not. The documents may come from teaching and research institutions in France or abroad, or from public or private research centers.
L'archive ouverte pluridisciplinaire HAL, est destinée au dépôt et à la diffusion de documents scientifiques de niveau recherche, publiés ou non, émanant des établissements d'enseignement et de recherche français ou étrangers, des laboratoires publics ou privés.

\section{(ㄷ)(1) $\$$}

Distributed under a Creative Commons Attribution - NonCommercial| 4.0 International 


\title{
A variational formulation of constitutive models and updates in non-linear finite viscoelasticity
}

\author{
Eduardo Fancello ${ }^{1, *, \dagger}$, Jean-Philippe Ponthot ${ }^{2, \dagger}$ and Laurent Stainier ${ }^{2, \S}, \mathbb{I}$ \\ ${ }^{1}$ Departmento de Engenharia Mecânica, Universidade Federal de Santa Catarina, Florianópolis, SC, Brazil \\ ${ }^{2}$ Dépt. AéroSpatiale, Mécanique et mAtériaux (ASMA/LTAS-MC\&T), Université de Liège, Belgium
}

The purpose of this article is to present a general framework for constitutive viscoelastic models in finite strain regime. The approach is qualified as variational since the constitutive updates obey a minimum principle within each load increment. The set of internal variables is strain-based and employs, according to the specific model chosen, a multiplicative decomposition of strain into elastic and viscous components. The present approach shares the same technical procedures used for analogous models of plasticity or viscoplasticity, such as the solution of a minimization problem to identify inelastic updates and the use of exponential mapping for time integration. However, instead of using the classical decomposition of inelastic strains into amplitude and direction, we take advantage of a spectral decomposition that provides additional facilities to accommodate, into simple analytical expressions, a wide set of specific models. Moreover, appropriate choices of the constitutive potentials allow the reproduction of other formulations in the literature. The final part of the paper presents a set of numerical examples in order to explore the characteristics of the formulation as well as its applicability to usual large-scale FEM analyses.

KEY WORDS: finite viscoelasticity; variational formulation; constitutive updates

\section{INTRODUCTION}

The main characteristic in viscoelastic models is the existence of a rate of deformations in the presence of non-zero (constant) states of stress. Moreover, inelastic strains occur in the

\footnotetext{
*Correspondence to: E. Fancello, GRANTE/EMC/UFSC, Campus Universitário-Trindade, 88040-900 Florianópolis, SC, Brazil.

†E-mail: fancello@grante.ufsc.br

${ }^{\ddagger}$ E-mail: JP.Ponthot@ulg.ac.be

${ }^{\S}$ E-mail: L.Stainier@ulg.ac.be

IIResearch Associate at the Belgian National Fund for Scientific Research (FNRS).
} 
presence of stresses, no matter what their intensity. This behaviour is in contrast with other models like plasticity or viscoplasticity, where a certain level of stress must be reached before inelastic deformations appear.

Many different models for viscoelastic materials in finite deformation regime are found in the literature. However, in contrast with what we see in small deformation models, the choice of convenient internal variables and evolution laws is neither trivial nor unique, leading to different formulations. Without claiming to perform a general classification, we would like to distinguish two possible approaches. From one side, we recall the work of Simo [1] in which an additive decomposition of stresses in equilibrium and non-equilibrium contributions is stated and, more relevant, the evolution law is defined as a linear differential equation based on the non-equilibrium stresses. This approach was later followed, among many others, by Holzapfel and Simo [2,3] and more recently by Holzapfel, Gasser and Bonet $[4,5]$. This last work modifies the original approach by including the well-known multiplicative split of isochoric deformation gradient into elastic and viscous parts and by defining the rate of nonequilibrium stress (i.e. the evolution equation) at constant total Cauchy deformation $\mathbf{C}$, which finally provides an evolution equation in terms of viscous Cauchy strain rate $\dot{\mathbf{C}}^{\mathrm{v}}$.

Multiplicative decomposition of strains applied to viscoelastic constitutive equations goes back to the work of Sidoroff [6] and later to References [7-9], among others. In Reference [10] the ability of different models to reproduce non-linear viscous behaviour is discussed and a model is proposed that is not restricted to small perturbations away from thermodynamic equilibrium. Based on a multiplicative decomposition of strain, the model is presented as an extension of the model proposed in Reference [7] for elastic deformation gradients $\mathbf{F}^{\mathrm{e}}$ far from the identity tensor.

The goal of this work is to provide a general framework for constitutive viscoelastic models based on the mathematical background proposed in References [11-13]. Thus, the approach is qualified as variational since the constitutive updates obey a minimum principle within each load increment. The set of internal variables is strain-based, and thus employs, according to the specific model chosen, multiplicative decomposition of strain into elastic and viscous components. In that sense, the present approach is closer to that of Reference [10] or the spatial model of Reference [5] than to that of Simo.

On the basis of the same theoretical framework, the present approach for viscoelastic models shares the same technical procedures shown in Reference [11] to deal with the local nonlinear constitutive problem, i.e. the solution of a minimization problem to identify inelastic updates. However, instead of using the classic decomposition of inelastic strains into amplitude and direction, we take profit of a spectral decomposition that provides additional facilities to accommodate, into simple analytical expressions, a wide set of viscous models. The only requirement is the definition of different isotropic potentials in terms of eigenvalues of strain and/or strain rates. As a consequence, general expressions for the stress updates as well as for the (symmetric) material tensor of the tangent matrices are obtained, which can be implemented independently of the particular potential and corresponding derivatives definitions. In addition, appropriate choices of the potentials retrieve other formulations in the literature such as, for example, the isotropic logarithmic-based model of Reference [5].

This article is organized as follows. Section 2 shows the formulation used for the balance problem. The general approach for irreversible constitutive problems is presented in Section 3, focusing later on the problem of viscoelastic materials, which is the main contribution of this paper (Sections 3.2-3.5). In Section 4 a set of numerical examples is shown in order to discuss 
the characteristics of the present approach. Finally, an Appendix includes technical mathematical operations that, for the purpose of clarity, were omitted in the main text.

\section{VARIATIONAL PRINCIPLE FOR BALANCE EQUATIONS}

We use this section to present a possible approach for the equilibrium problem. Using conventional notation, let us call

$$
\mathbf{F}=\nabla_{0} \mathbf{x}, \quad \mathbf{C}=\mathbf{F}^{\mathrm{T}} \mathbf{F}, \quad \mathbf{B}=\mathbf{F F}^{\mathrm{T}}
$$

the gradient of deformations, the Cauchy strain tensor and the Finger strain tensor, respectively. In order to circumvent locking in isoparametrical finite elements, the equilibrium will be stated using the variational formulation proposed in Reference [14]. In this approach, the pressure field $\pi$ and volumetric strain field $\theta$ are assumed to be constant over each element. A volumetric/isochoric split is performed and the following kinematic tensors are defined:

$$
\begin{aligned}
& \hat{\mathbf{F}}=\frac{1}{J^{1 / 3}} \mathbf{F}, \quad J=\operatorname{det}(\mathbf{F}), \quad \hat{\mathbf{C}}=\hat{\mathbf{F}}^{\mathrm{T}} \hat{\mathbf{F}}, \quad \hat{\mathbf{B}}=\hat{\mathbf{F}} \hat{\mathbf{F}}^{\mathrm{T}} \\
& \overline{\mathbf{F}}=\left(\frac{\theta}{J}\right)^{1 / 3} \mathbf{F}=\theta^{1 / 3} \hat{\mathbf{F}}, \quad \overline{\mathbf{C}}=\left(\frac{\theta}{J}\right)^{2 / 3} \quad \mathbf{C}=\theta^{2 / 3} \hat{\mathbf{C}}
\end{aligned}
$$

Let us assume a hyperelastic material for which the internal free energy $\Psi$ allows an additive decomposition in volumetric and isochoric parts:

$$
\Psi(\overline{\mathbf{C}})=W(\hat{\mathbf{C}})+U(\theta)
$$

The three-field functional $\mathscr{H}[x, \theta, \pi]$ is defined as

$$
\mathscr{H}[x, \theta, \pi]=\sum_{e=1}^{E} \int_{\Omega_{0}} \Psi(\overline{\mathbf{C}})+\pi(J-\theta) \mathrm{d} \Omega_{0}-T F_{\mathrm{e}}(x)
$$

where $T F_{\mathrm{e}}(x)$ is the work of external forces. This functional can also be stated incrementally, i.e. for given values $\left[x_{n}, \theta_{n}, \pi_{n}\right]$ it is possible to write a potential of the type

$$
\mathscr{H}\left[x_{n+1}, \theta_{n+1}, \pi_{n+1}\right]=\sum_{e=1}^{E} \int_{\Omega_{0}} \Psi\left(\overline{\mathbf{C}}_{n+1}\right)+\pi_{n+1}\left(J_{n+1}-\theta_{n+1}\right) \mathrm{d} \Omega_{0}-T F_{\mathrm{e}}\left(x_{n+1}\right)
$$

whose stationarity conditions define the value of $\theta$ and $\pi$ as well as the balance equation

$$
\begin{aligned}
\left\langle D_{\varphi_{n+1}} \mathscr{H}, \delta x\right\rangle & =\sum_{e=1}^{E} \int_{\Omega_{0}} \overline{\mathbf{P}}_{n+1} \cdot \nabla_{0} \delta x \mathrm{~d} \Omega_{0}-T F_{\mathrm{e}}\left(x_{n+1}\right)=0 \\
\overline{\mathbf{P}}_{n+1} & =\mathbf{F}_{n+1}\left[J_{n+1}^{-2 / 3} \operatorname{DEV}\left(2 \frac{\partial W}{\partial \hat{\mathbf{C}}_{n+1}}\right)+\frac{\partial U}{\partial \theta_{n+1}} J_{n+1} \mathbf{C}_{n+1}^{-1}\right]
\end{aligned}
$$


where $\operatorname{DEV}(\mathbf{A})=\mathbf{A}-\frac{1}{3}(\mathbf{A}: \mathbf{C}) \mathbf{C}^{-1}$. The last expression provides stress recovery of the conventional first Piola-Kirchhoff stress tensor. It is worth noting that the expression of $\overline{\mathbf{P}}_{n+1}$ is of the hyperelastic type in the sense that the stress is derived from a potential function. In (7) this relationship is given incrementally. It will be seen in the next sections that incremental dissipative models can be described using incremental potential functions $\Psi\left(\overline{\mathbf{C}}_{n+1}\right)$. In other words, dissipative models can be treated as 'hyperelastic' within each increment. In this case, $\Psi\left(\overline{\mathbf{C}}_{n+1}\right)$ is usually referred to as a hyperelastic 'pseudo-potential'.

\section{CONSTITUTIVE PROBLEM}

\subsection{Variational formulation for inelastic materials}

The following lines describe a general variational formulation for inelastic materials subjected to finite strains. Although these ideas are originally proposed in Reference [11], their main outlines will be repeated with the aim of keeping this article self-contained. This approach is completely imbedded within the more general framework of internal-variable continuum thermodynamics. Thus, the first step to describe a constitutive behaviour is to define a general set $\mathscr{E}$ of external and internal variables:

$$
\mathscr{E}=\left\{\mathbf{F}, \mathbf{F}^{\mathrm{i}}, \mathbf{Q}\right\}
$$

where a multiplicative decomposition of the gradient of deformations is assumed and $\mathbf{F}^{\mathrm{i}}$ is the inelastic part of the (total) deformation $\mathbf{F}=\mathbf{F}^{\mathrm{e}} \mathbf{F}^{\mathrm{i}}$. Moreover, $\mathbf{Q}$ contains all the remaining internal variables of the model. Consider the existence of a free energy potential $W$ and a dissipative potential $\phi$,

$$
W(\mathscr{E})=\varphi(\mathbf{F})+\varphi^{\mathrm{e}}\left(\mathbf{F F}^{\mathrm{i}-1}\right)+\varphi^{\mathrm{i}}\left(\mathbf{F}^{\mathrm{i}}, \mathbf{Q}\right), \quad \phi=\phi(\dot{\mathbf{F}}, \mathscr{E})
$$

such that the Piola-Kirchhoff stress tensor and conjugate forces are defined as

$$
\mathbf{P}=\frac{\partial W}{\partial \mathbf{F}}(\mathscr{E})+\frac{\partial \phi}{\partial \dot{\mathbf{F}}}(\dot{\mathbf{F}} ; \mathscr{E}), \quad \mathbf{T}=-\frac{\partial W}{\partial \mathbf{F}^{\mathrm{i}}}(\mathscr{E}), \quad \mathbf{A}=-\frac{\partial W}{\partial \mathbf{Q}}(\mathscr{E})
$$

In addition, we assume the existence of a dissipative potential $\psi$ such that the relations between the local irreversible process and the local state are implicitly given by

$$
\begin{aligned}
& \psi=\psi\left(\dot{\mathbf{F}}^{\mathrm{i}}, \dot{\mathbf{Q}} ; \mathscr{E}\right) \\
& \mathbf{T}=\frac{\partial \psi}{\partial \dot{\mathbf{F}}^{\mathrm{i}}}\left(\dot{\mathbf{F}}^{\mathrm{i}}, \dot{\mathbf{Q}} ; \mathscr{E}\right), \quad \mathbf{A}=\frac{\partial \psi}{\partial \dot{\mathbf{Q}}^{\mathrm{i}}}\left(\dot{\mathbf{F}}^{\mathrm{i}}, \dot{\mathbf{Q}} ; \mathscr{E}\right)
\end{aligned}
$$

The potential $\psi$ was defined independently of $\phi$ in order to introduce a dissipation term depending only on the inelastic deformation rate $\dot{\mathbf{F}}^{i}$ (note that $\phi$ depends on the total rate $\dot{\mathbf{F}}$ ). The viscoelastic formulation proposed in the next sections will use this distinction.

From (10) and (12) it is clear that, at any state $\mathscr{E}$,

$$
\frac{\partial W}{\partial \mathbf{F}^{\mathrm{i}}}+\frac{\partial \psi}{\partial \dot{\mathbf{F}}^{\mathrm{i}}}=0, \quad \frac{\partial W}{\partial \mathbf{Q}}+\frac{\partial \psi}{\partial \dot{\mathbf{Q}}}=0
$$


which are constraints that must be satisfied by the rate variables $\dot{\mathbf{F}}^{\mathrm{i}}$ and $\dot{\mathbf{Q}}$. The satisfaction of (13) is equivalent to finding, for a given state $\mathscr{E}$, the values $\dot{\mathbf{F}}^{\mathrm{i}}$ and $\dot{\mathbf{Q}}$ that solve the minimization problem:

$$
\min _{\dot{\mathbf{F}}^{\mathrm{i}}, \dot{\mathbf{Q}}}\left\{\frac{\partial W(\mathscr{E})}{\partial \mathbf{F}^{\mathrm{i}}} \cdot \dot{\mathbf{F}}^{\mathrm{i}}+\frac{\partial W(\mathscr{E})}{\partial \mathbf{Q}} \dot{\mathbf{Q}}+\psi\left(\dot{\mathbf{F}}^{\mathrm{i}}, \dot{\mathbf{Q}} ; \mathscr{E}\right)\right\}
$$

A convenient new potential is now defined:

$$
\begin{aligned}
\mathscr{D}\left(\dot{\mathbf{F}}, \dot{\mathbf{F}}^{\mathrm{i}}, \dot{\mathbf{Q}} ; \mathscr{E}\right) & =\frac{\partial W(\mathscr{E})}{\partial \mathbf{F}} \cdot \dot{\mathbf{F}}+\frac{\partial W(\mathscr{E})}{\partial \mathbf{F}^{\mathrm{i}}} \cdot \dot{\mathbf{F}}^{\mathrm{i}}+\frac{\partial W(\mathscr{E})}{\partial \mathbf{Q}} \dot{\mathbf{Q}}+\phi(\dot{\mathbf{F}}, \mathscr{E})+\psi\left(\dot{\mathbf{F}}^{\mathrm{i}}, \dot{\mathbf{Q}} ; \mathscr{E}\right) \\
& =\frac{\partial W(\mathscr{E})}{\partial \mathscr{E}} \dot{\mathscr{E}}+\phi(\dot{\mathbf{F}}, \mathscr{E})+\psi\left(\dot{\mathbf{F}}^{\mathrm{i}}, \dot{\mathbf{Q}} ; \mathscr{E}\right)
\end{aligned}
$$

with the following characteristics:

- Its minimization with respect to the inelastic rate variables $\dot{\mathbf{F}}^{\mathrm{i}}, \dot{\mathbf{Q}}$ provides the values $\dot{\mathbf{F}}^{\text {i* }}, \dot{\mathbf{Q}}^{*}$ that satisfy the constraint (13) or, equivalently, (14).

- Its derivative with respect to the rate variable $\dot{\mathbf{F}}$ provides the constitutive state equation for $\mathbf{P}$ (see (10)).

The minimization on $\dot{\mathbf{F}}^{\mathrm{i}}, \dot{\mathbf{Q}}$ defines a new functional, having $\dot{\mathbf{F}}$ as a variable:

$$
\mathscr{D}_{\mathrm{eff}}(\dot{\mathbf{F}} ; \mathscr{E})=\phi(\dot{\mathbf{F}}, \mathscr{E})+\min _{\dot{\mathbf{F}}^{\mathrm{i}}, \dot{\mathbf{Q}}}\left\{\frac{\partial W(\mathscr{E})}{\partial \mathbf{F}} \cdot \dot{\mathbf{F}}+\frac{\partial W(\mathscr{E})}{\partial \mathbf{F}^{i}} \cdot \dot{\mathbf{F}}^{\mathrm{i}}+\frac{\partial W(\mathscr{E})}{\partial \mathbf{Q}} \dot{\mathbf{Q}}+\psi\left(\dot{\mathbf{F}}^{\mathrm{i}}, \dot{\mathbf{Q}} ; \mathscr{E}\right)\right\}
$$

that, as already remarked, has the following property:

$$
\frac{\partial \mathscr{D}_{\mathrm{eff}}(\dot{\mathbf{F}} ; \mathscr{E})}{\partial \dot{\mathbf{F}}}=\frac{\partial W(\mathscr{E})}{\partial \mathbf{F}}+\frac{\partial \phi}{\partial \dot{\mathbf{F}}}(\dot{\mathbf{F}} ; \mathscr{E})=\mathbf{P}
$$

Thus, $\mathscr{D}_{\text {eff }}(\dot{\mathbf{F}} ; \mathscr{E})$ constitutes a rate potential of $\dot{\mathbf{F}}$ for $\mathbf{P}$.

The next step is to define an incremental constitutive equation within the interval $\left[t_{n}, t_{n+1}\right]$ that provides incremental updates $\mathbf{F}_{n+1}, \dot{\mathbf{F}}_{n+1}, \mathbf{Q}_{n+1}$ consistent with the rate potential (17). The main objective is to obtain an incremental potential $\Psi\left(\mathbf{F}_{n+1}\right)$ such that due to objectivity constraints [15-17],

$$
\begin{aligned}
\mathbf{P}_{n+1} & =\frac{\partial \Psi\left(\mathbf{F}_{n+1}\right)}{\partial \mathbf{F}_{n+1}}=2 \mathbf{F}_{n+1} \frac{\partial \Psi\left(\mathbf{C}_{n+1}\right)}{\partial \mathbf{C}_{n+1}} \\
& =\mathbf{F}_{n+1}\left[J_{n+1}^{-2 / 3} \operatorname{DEV}\left(2 \frac{\partial W}{\partial \hat{\mathbf{C}}_{n+1}}\right)+\frac{\partial U}{\partial \theta_{n+1}} J_{n+1} \mathbf{C}_{n+1}^{-1}\right]
\end{aligned}
$$

This means that the internal variables $\mathbf{F}_{n}^{\mathrm{i}}{ }_{1}$ and $\mathbf{Q}_{n+1}$ can be computed by the incremental constitutive equations once the value of $\mathbf{F}_{n}^{+}+1$ is known. A possible general expression for the 


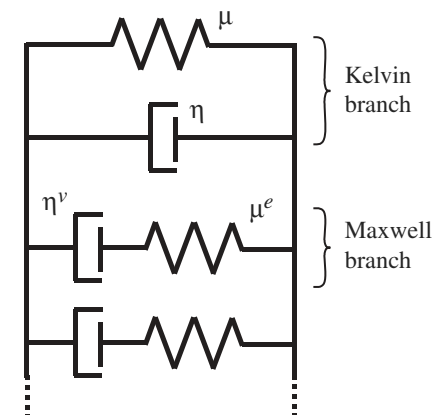

Figure 1. Generalized Kelvin-Maxwell model.

potential is

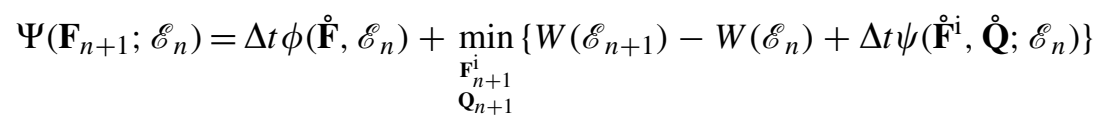

where $\stackrel{\circ}{\mathbf{F}}\left(\mathbf{F}_{n+1}, \mathscr{E}_{n}\right), \stackrel{\circ}{\mathbf{F}}^{\mathrm{i}}\left(\mathbf{F}_{n+1}^{\mathrm{i}}, \mathscr{E}_{n}\right)$ and $\stackrel{\mathbf{Q}}{ }\left(\mathbf{Q}_{n+1}, \mathscr{E}_{n}\right)$ are suitable incremental approximations of the rate variables $\dot{\mathbf{F}}, \dot{\mathbf{F}}^{i}$, and $\dot{\mathbf{Q}}$, respectively. It is possible to show that if $\Delta t \rightarrow 0$, this incremental potential reduces to (16), which is the claimed consistence for the constitutive update. A final comment is necessary to close the gap between constitutive and balance equations. If, due to numerical implementation, the variational principle (5) is used, then (20) plays the role of incremental potential whose derivative given by (19) provides the new value for the stress tensor. This will be seen more clearly in the next sections.

\subsection{A group of visco-hyperelastic models}

A quite general group of viscoelastic materials can be modelled within the present variational framework. Owing to the possibility of obtaining analytical or semi-analytical expressions for the constitutive updates, only isotropic models will be considered now. However, no theoretical limitations to include more general non-isotropic behaviours are found.

The rheological mechanism shown in Figure 1 is taken as a basis to include different potential expressions in (16) or (20). The model is based on the following assumptions:

- The elastic part of the Kelvin branch accounts for a separation of the elastic energy into isochoric and volumetric parts. The isochoric part is an isotropic function of $\hat{\mathbf{C}}=\hat{\mathbf{F}}^{\mathrm{T}} \hat{\mathbf{F}}$ :

$$
\varphi(\hat{\mathbf{C}})=\varphi\left(c_{1}, c_{2}, c_{3}\right)
$$

where $c_{j}$ and $\mathbf{E}_{j}$ are the eigenvalues and eigenvectors of $\hat{\mathbf{C}}$ The volumetric part may be defined using the usual expression

$$
U(\theta)=\frac{K}{2}[\ln \theta]^{2}
$$

where $K$ is the bulk modulus of the material. 
- Analogously, the viscous part of the Kelvin branch is also an isotropic function of the symmetric part of the rate of deformation:

$$
\begin{aligned}
\mathbf{D} & =\operatorname{dev}\left[\operatorname{Sym}\left(\dot{\mathbf{F}} \mathbf{F}^{-1}\right)\right] \\
\phi(\mathbf{D}) & =\phi\left(d_{1}, d_{2}, d_{3}\right)
\end{aligned}
$$

where $d_{j}$ and $\mathbf{M}_{j}$ are, respectively, the eigenvalues and eigenvectors of $\mathbf{D}$.

- The Maxwell branch, connected in parallel, takes into account the multiplicative separation of elastic and inelastic (viscous) strains. The viscous part is assumed to be isochoric:

$$
\hat{\mathbf{F}}=\hat{\mathbf{F}}^{\mathrm{e}} \mathbf{F}^{\mathrm{v}} \Longrightarrow \hat{\mathbf{F}}^{\mathrm{e}}=\hat{\mathbf{F}} \mathbf{F}^{\mathrm{v}-1}, \quad \operatorname{det} \mathbf{F}^{\mathrm{v}}=1
$$

A viscous rate of deformation (or viscous stretching) $\mathbf{D}^{\mathrm{v}}$ is defined as

$$
\mathbf{D}^{\mathrm{v}}=\operatorname{Sym}\left(\mathbf{L}^{\mathrm{v}}\right)=\mathbf{L}^{\mathrm{v}}=\dot{\mathbf{F}}^{\mathrm{v}} \mathbf{F}^{\mathrm{v}-1}
$$

which, by construction, is deviatoric. The symmetry of $\mathbf{L}^{\mathrm{V}}$ comes from the null viscous spin assumption ( $\operatorname{skew}\left(\mathbf{L}^{\mathrm{v}}\right)=\mathbf{W}^{\mathrm{v}}=0$ ). Rearranging (26) a flow rule for the internal variable $\mathbf{F}^{\mathrm{v}}$ is obtained as

$$
\dot{\mathbf{F}}^{\mathrm{v}}=\mathbf{D}^{\mathrm{v}} \mathbf{F}^{\mathrm{v}}
$$

- Additional constraints on $\mathbf{D}^{\mathrm{v}}$ define specific characteristics of the flow rule. In plastic or viscoplastic von Mises models, the normality rule constrains $\mathbf{D}^{\mathrm{v}}$ to be of type

$$
\begin{aligned}
\mathbf{D}^{\mathrm{v}} & =d^{\mathrm{v}} \mathbf{M}^{\mathrm{v}} \\
d^{\mathrm{v}} & \in \mathbb{R}^{+} \\
\mathbf{M}^{\mathrm{v}} \in K_{M} & =\left\{\mathbf{N} \in \operatorname{Sym}: \mathbf{N} \cdot \mathbf{N}=\frac{3}{2}, \mathbf{N} \cdot \mathbf{I}=0\right\}
\end{aligned}
$$

where I is the second-order identity matrix, Sym represents the set of symmetric secondorder tensors and $\mathbf{N}$ a traceless symmetric tensor. The scalar $d^{\mathrm{v}}$ accounts for the amplitude of $\mathbf{D}^{\mathrm{v}}$ while $\mathbf{M}^{\mathrm{v}}$ provides normality and tracelessness properties. A different and convenient decomposition is proposed here:

$$
\begin{aligned}
\mathbf{D}^{\mathrm{v}} & =\sum_{i=1}^{3} d_{j}^{\mathrm{v}} \mathbf{M}_{j}^{\mathrm{v}} \\
d^{\mathrm{v}} \in K_{Q} & =\left\{p_{j} \in \mathbb{R}: p_{1}+p_{2}+p_{3}=0\right\} \\
\mathbf{M}_{j}^{\mathrm{v}} \in K_{M} & =\left\{\mathbf{N}_{j} \in \operatorname{Sym}: \mathbf{N}_{j} \cdot \mathbf{N}_{j}=1, \quad \mathbf{N}_{i} \cdot \mathbf{N}_{j}=0, \quad i \neq j\right\}
\end{aligned}
$$

where $d_{j}^{\mathrm{v}}$ and $\mathbf{M}_{j}^{\mathrm{v}}, j=1,2,3$, are eigenvalues and eigenprojections of $\mathbf{D}^{\mathrm{v}}$. The tracelessness condition is enforced by the set $K_{Q}$ while $K_{M}$ constrains eigenprojections to be symmetric orthonormal second-order tensors. Scalars $d_{j}^{\mathrm{v}}$ account for the amplitude of viscous (inelastic) stretching and, consequently, they are chosen to be the internal variables contained in the set $\dot{\mathbf{Q}}=\left\{d_{1}, d_{2}, d_{3}\right\}$. In this case, it is important to note that (27) 
is a non-holonomic constraint relating the internal variables $\mathbf{F}^{\mathbf{v}}$ and $\mathbf{Q}$. The elastic and viscous potentials associated with this branch are assumed to be isotropic functions of the elastic deformation and viscous stretching, and thus they depend on their corresponding eigenvalues:

$$
\begin{aligned}
\varphi^{\mathrm{e}}\left(\hat{\mathbf{C}}^{\mathrm{e}}\right) & =\varphi^{\mathrm{e}}\left(c_{1}^{\mathrm{e}}, c_{2}^{\mathrm{e}}, c_{3}^{\mathrm{e}}\right) \\
\psi\left(\mathbf{D}^{\mathrm{v}}\right) & =\psi\left(d_{1}^{\mathrm{v}}, d_{2}^{\mathrm{v}}, d_{3}^{\mathrm{v}}\right)
\end{aligned}
$$

where $c_{j}^{\mathrm{e}}$ and $\mathbf{E}_{j}^{\mathrm{e}}$ are the eigenvalues and eigenvectors of $\hat{\mathbf{C}}^{\mathrm{e}}$ :

$$
\hat{\mathbf{C}}^{\mathrm{e}}=\hat{\mathbf{F}}^{\mathrm{e} \mathrm{T}} \hat{\mathbf{F}}^{\mathrm{e}}=\sum_{j=1}^{3} c_{j}^{\mathrm{e}} \mathbf{E}_{j}^{\mathrm{e}}
$$

- Viscous deformations are incrementally updated based on exponential mappings:

$$
\begin{aligned}
\Delta \hat{\mathbf{F}} & =\hat{\mathbf{F}}_{n+1} \hat{\mathbf{F}}_{n}^{-1}=\Delta \mathbf{R}(\exp [\Delta t \mathbf{D}]) \\
\Delta t \mathbf{D} & =\Delta t\left(d_{j} \mathbf{M}_{j}\right)=\sum_{j=1}^{3} \Delta q_{j} \mathbf{M}_{j} \\
\Delta \hat{\mathbf{C}} & =(\Delta \hat{\mathbf{F}})^{\mathrm{T}} \Delta \hat{\mathbf{F}}=\hat{\mathbf{F}}_{n}^{-\mathrm{T}} \hat{\mathbf{C}}_{n+1} \hat{\mathbf{F}}_{n}^{-1}=\exp [\Delta t \mathbf{D}]^{2} \\
\Rightarrow \mathbf{D} & =\sum_{j=1}^{3} \frac{\Delta q_{j}}{\Delta t} \mathbf{M}_{j}=\frac{1}{2 \Delta t} \ln (\Delta \hat{\mathbf{C}})
\end{aligned}
$$

where $\Delta \mathbf{R}$ comes from the polar decomposition of $\Delta \hat{\mathbf{F}}$ (note that it is later cancelled in the calculation of $\Delta \hat{\mathbf{C}})$. In analogous form,

$$
\begin{aligned}
\Delta \mathbf{F}^{\mathrm{v}} & =\mathbf{F}_{n+1}^{\mathrm{v}} \mathbf{F}_{n}^{\mathrm{v}-1}=\exp \left[\Delta t \mathbf{D}^{\mathrm{v}}\right] \\
\Delta t \mathbf{D}^{\mathrm{v}} & =\Delta t\left(d_{j}^{\mathrm{v}} \mathbf{M}_{j}^{\mathrm{v}}\right)=\sum_{j=1}^{3} \Delta q_{j}^{\mathrm{v}} \mathbf{M}_{j}^{\mathrm{v}} \\
\Delta \mathbf{C}^{\mathrm{v}} & =\left(\Delta \mathbf{F}^{\mathrm{v}}\right)^{\mathrm{T}} \Delta \mathbf{F}^{\mathrm{v}}=\mathbf{F}_{n}^{\mathrm{v}-\mathrm{T}} \mathbf{C}_{n+1}^{\mathrm{v}} \mathbf{F}_{n}^{\mathrm{v}-1}=\exp \left[\Delta t \mathbf{D}^{\mathrm{v}}\right]^{2} \\
\Rightarrow \mathbf{D}^{\mathrm{v}} & =\sum_{j=1}^{3} \frac{\Delta q_{j}^{\mathrm{v}}}{\Delta t} \mathbf{M}_{j}^{\mathrm{v}}=\frac{1}{2 \Delta t} \ln \left(\Delta \mathbf{C}^{\mathrm{v}}\right)
\end{aligned}
$$

Expressions (40) and (44) show that $\mathbf{D}$ and $\mathbf{D}^{\mathrm{v}}$ are approximated by incremental expressions of $\Delta \hat{\mathbf{C}}$ and $\Delta \mathbf{C}^{\mathrm{v}}$, respectively. The exponential mapping has the particularly convenient property of providing an isochoric tensor for any traceless argument $[18,19]$. 


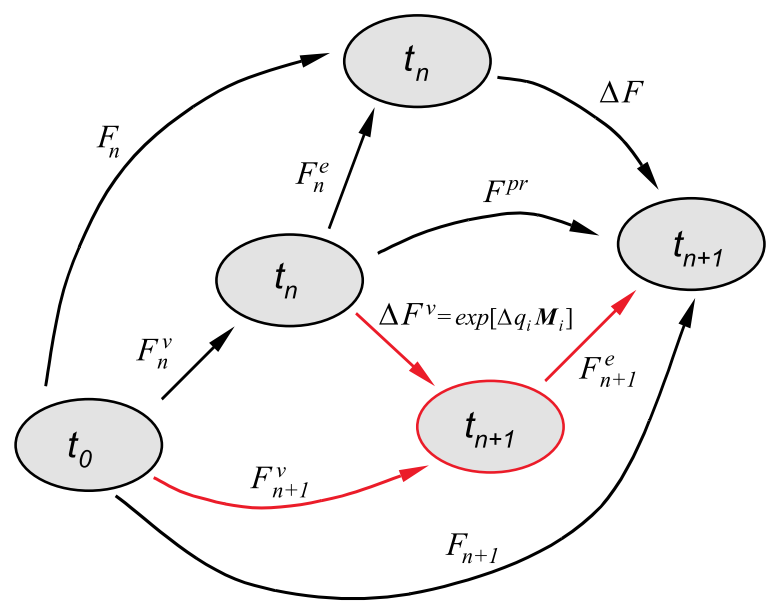

Figure 2. Multiplicative strain decomposition.

Quantities $\Delta \mathbf{F}^{\mathrm{v}}$ and $\Delta \hat{\mathbf{F}}$ are represented in Figure 2 that shows the strain decomposition considered in the present approach.

Owing to the inclusion of constraints (40) and (44) in (20), the minimizing variables $\mathbf{Q}_{n+1}, \mathbf{F}_{n+1}^{\mathrm{v}}$ are substituted by the new incremental variables $\Delta q_{j}^{\mathrm{v}}, \mathbf{M}_{j}^{\mathrm{v}}$. The minimization problem is thus re-written as

$$
\begin{aligned}
\Psi\left(\mathbf{F}_{n+1} ; \mathscr{E}_{n}\right)= & \Psi\left(\mathbf{C}_{n+1} ; \mathscr{E}_{n}\right)=\Delta \varphi\left(\hat{\mathbf{C}}_{n+1}\right)+\Delta t \phi\left(\frac{\Delta q_{j}^{\mathrm{v}}}{\Delta t}\right)+\Delta U\left(\theta_{n+1}\right) \\
& +\min _{\mathbf{M}_{j}^{\mathrm{v}}, \Delta q_{j}^{\mathrm{v}}}\left\{\Delta \varphi^{\mathrm{e}}\left(\hat{\mathbf{C}}_{n+1}^{\mathrm{e}}\right)+\Delta t \psi\left(\frac{\Delta q_{j}^{\mathrm{v}}}{\Delta t}\right)\right\} \\
\Delta \varphi\left(\hat{\mathbf{C}}_{n+1}\right)= & \varphi\left(\hat{\mathbf{C}}_{n+1}\right)-\varphi\left(\hat{\mathbf{C}}_{n}\right) \\
\Delta \varphi^{\mathrm{e}}\left(\hat{\mathbf{C}}_{n+1}^{\mathrm{e}}\right)= & \varphi\left(\hat{\mathbf{C}}_{n+1}^{\mathrm{e}}\right)-\varphi\left(\hat{\mathbf{C}}_{n}^{\mathrm{e}}\right) \\
\Delta U\left(\theta_{n+1}\right)= & U\left(\theta_{n+1}\right)-U\left(\theta_{n}\right)
\end{aligned}
$$

such that

$$
\begin{aligned}
& \Delta q_{j}^{\mathrm{v}} \in K_{Q}=\left\{p_{j} \in \mathbb{R}^{1}: p_{1}+p_{2}+p_{3}=0\right\} \\
& \mathbf{M}_{j}^{\mathrm{v}} \in K_{M}=\left\{\mathbf{N}_{j} \in \operatorname{Sym}: \mathbf{N}_{j} \cdot \mathbf{N}_{j}=1, \quad \mathbf{N}_{i} \cdot \mathbf{N}_{j}=0, i \neq j\right\}
\end{aligned}
$$

The set $K_{Q}$ enforces the traceless form of $\mathbf{D}^{\mathrm{v}}$, while the set $K_{M}$ accounts for usual properties of eigenprojections. Moreover, it is easy to verify that both sets are convex on their respective variables. 
Assuming convenient convexity properties for the terms within braces in (45), first-order optimality conditions are sufficient to obtain the minimizing arguments, i.e. to find $\Delta q_{j}^{\mathrm{v}} \in K_{Q}$ and $\mathbf{M}_{j}^{\mathrm{v}} \in K_{M}$ such that

$$
\begin{array}{cl}
\frac{\partial \Psi\left(\Delta q_{j}^{\mathrm{v}}, \mathbf{M}_{j}^{\mathrm{v}}\right)}{\partial \mathbf{M}_{j}^{\mathrm{v}}}[\delta \mathbf{M}]=0 & \forall \delta \mathbf{M} \in K_{M} \\
\frac{\partial \Psi\left(\Delta q_{j}^{\mathrm{v}}, \mathbf{M}_{j}^{\mathrm{v}}\right)}{\partial \Delta q_{j}^{\mathrm{v}}}[\delta q]=0 & \forall \delta q \in K_{Q}
\end{array}
$$

Taking into account the isotropy property of the energy functions, the minimization in (45) can be performed analytically. To this aim, an important relation between elastic and inelastic (viscous) deformations must be shown:

$$
\begin{aligned}
& \hat{\mathbf{F}}_{n+1}^{\mathrm{e}}=\hat{\mathbf{F}}_{n+1} \hat{\mathbf{F}}_{n+1}^{\mathrm{v}-1}=\hat{\mathbf{F}}^{\mathrm{pr}}\left(\exp \left[\Delta t \mathbf{D}^{\mathrm{v}}\right]\right)^{-1}, \quad \hat{\mathbf{F}}^{\mathrm{pr}}=\hat{\mathbf{F}}_{n+1} \mathbf{F}_{n}^{\mathrm{v}-1} \\
& \hat{\mathbf{C}}_{n+1}^{\mathrm{e}}=\hat{\mathbf{F}}_{n+1}^{\mathrm{eT}} \hat{\mathbf{F}}_{n+1}^{\mathrm{e}}=\hat{\mathbf{C}}^{\mathrm{pr}}\left(\exp \left[\Delta t \mathbf{D}^{\mathrm{v}}\right]\right)^{-2}, \quad \hat{\mathbf{C}}^{\mathrm{pr}}=\mathbf{F}_{n}^{\mathrm{v}-\mathrm{T}} \hat{\mathbf{C}}_{n+1} \mathbf{F}_{n}^{\mathrm{v}-1} \\
& \varepsilon_{n+1}^{\mathrm{e}}=\frac{1}{2} \ln \hat{\mathbf{C}}_{n+1}^{\mathrm{e}}=\varepsilon^{\mathrm{pr}}-\Delta t \mathbf{D}^{\mathrm{v}}, \quad \varepsilon^{\mathrm{pr}}=\frac{1}{2} \ln \hat{\mathbf{C}}^{\mathrm{pr}}
\end{aligned}
$$

In Equation (54), the assumption of co-linearity between $\hat{\mathbf{C}}^{\mathrm{pr}}$ and $\mathbf{D}^{\mathrm{v}}$ was taken into account to allow permutation between both tensors. This means that $\hat{\mathbf{C}}_{n+1}^{\mathrm{e}}, \hat{\mathbf{C}}^{\mathrm{pr}}$ and $\mathbf{D}^{\mathrm{v}}$ share the same eigenvectors $\mathbf{E}_{j}^{\mathrm{e}}=\mathbf{E}_{j}^{\mathrm{pr}}=\mathbf{M}_{j}^{\mathrm{v}}$ (we must remember that co-linearity does not mean proportionality). Substituting generic expressions (34), (35) and result (55) in condition (51), the co-linearity condition is entirely verified (see Appendix for related details). Finally, the satisfaction of (52) provides a set of non-linear equations for $\Delta q_{1}^{\mathrm{v}}, \Delta q_{2}^{\mathrm{v}}, \Delta q_{3}^{\mathrm{v}}$ :

$$
\begin{aligned}
\frac{\partial \varphi^{\mathrm{e}}}{\partial \varepsilon_{j}^{\mathrm{e}}}-\frac{\partial \psi}{\partial d_{j}^{\mathrm{v}}}+\lambda & =0, \quad j=1,2,3 \\
\Delta q_{1}^{\mathrm{v}}+\Delta q_{2}^{\mathrm{v}}+\Delta q_{3}^{\mathrm{v}} & =0
\end{aligned}
$$

where $\lambda$ is a Lagrangian multiplier and $\varepsilon_{j}^{\mathrm{e}}=\ln \left(c_{j}^{\mathrm{e}}\right) / 2$ are the eigenvalues of $\varepsilon_{n+1}^{\mathrm{e}}$.

Equivalently, due to the linear relation $\varepsilon_{j}^{\mathrm{e}}=\varepsilon_{j}^{\mathrm{pr}}-\Delta q_{j}^{\mathrm{v}}$, optimality conditions (56), (57) can be re-written as a non-linear function of $\varepsilon_{1}^{\mathrm{e}}, \varepsilon_{2}^{\mathrm{e}}, \varepsilon_{3}^{\mathrm{e}}$ (this will later prove to be advantageous to obtain second derivatives of $\varphi^{\mathrm{e}}$, see Appendix):

$$
\begin{gathered}
\frac{\partial \varphi^{\mathrm{e}}}{\partial \varepsilon_{j}^{\mathrm{e}}}-\frac{\partial \psi}{\partial d_{j}^{\mathrm{v}}}+\lambda=0, \quad j=1,2,3 \\
\varepsilon_{1}^{\mathrm{e}}+\varepsilon_{2}^{\mathrm{e}}+\varepsilon_{3}^{\mathrm{e}}=0
\end{gathered}
$$

The existence of a solution for this system is guaranteed if convexity properties are assumed for both $\varphi^{\mathrm{e}}$ and $\psi$. 
Once the minimization is performed, the derivative of $\Psi$ with respect to $\hat{\mathbf{C}}_{n+1}$ and $\theta_{n+1}$ should be calculated in order to obtain the Piola-Kirchhoff stress tensor. The deviatoric part is given by (see Appendix for operational details)

$$
\begin{aligned}
\frac{\partial \varphi}{\partial \hat{\mathbf{C}}_{n+1}} & =\sum_{j=1}^{3} \frac{\partial \varphi}{\partial c_{j}} \frac{\partial c_{j}}{\partial \hat{\mathbf{C}}_{n+1}}=\sum_{j=1}^{3} \frac{\partial \varphi}{\partial c_{j}} \mathbf{E}_{j} \\
\frac{\partial \phi}{\partial \hat{\mathbf{C}}_{n+1}} & =\left(\sum_{j=1}^{3} \frac{\partial \varphi}{\partial d_{j}} \frac{\partial d_{j}}{\partial \Delta c_{j}} \frac{\partial \Delta c_{j}}{\partial \Delta \hat{\mathbf{C}}}\right) \frac{\partial \Delta \hat{\mathbf{C}}}{\partial \hat{\mathbf{C}}_{n+1}}=\hat{\mathbf{F}}_{n}^{-1}\left(\sum_{j=1}^{3} \frac{\partial \phi}{\partial d_{j}} \frac{1}{2 \Delta t \Delta c_{j}} \mathbf{M}_{j}\right) \hat{\mathbf{F}}_{n}^{-\mathrm{T}} \\
\frac{\partial \varphi^{\mathrm{e}}}{\partial \hat{\mathbf{C}}_{n+1}} & =\left(\sum_{j=1}^{3} \frac{\partial \varphi^{\mathrm{e}}}{\partial c_{j}^{\mathrm{e}}} \frac{\partial c_{j}^{\mathrm{e}}}{\partial \hat{\mathbf{C}}_{n+1}^{\mathrm{e}}}\right) \frac{\partial \hat{\mathbf{C}}_{n+1}^{\mathrm{e}}}{\partial \hat{\mathbf{C}}_{n+1}}=\mathbf{F}_{n+1}^{\mathrm{v}-1}\left(\sum_{j=1}^{3} \frac{\partial \varphi^{\mathrm{e}}}{\partial \varepsilon_{j}^{\mathrm{e}}} \frac{1}{2 c_{j}^{\mathrm{e}}} \mathbf{E}_{j}^{\mathrm{e}}\right) \mathbf{F}_{n+1}^{\mathrm{v}-\mathrm{T}} \\
& =\left(\sum_{j=1}^{3} \frac{\partial \varphi^{\mathrm{e}}}{\partial c_{j}^{\mathrm{pr}}} \frac{\partial c_{j}^{\mathrm{pr}}}{\partial \hat{\mathbf{C}}^{\mathrm{pr}}}\right) \frac{\partial \hat{\mathbf{C}}^{\mathrm{pr}}}{\partial \hat{\mathbf{C}}_{n+1}}=\mathbf{F}_{n}^{\mathrm{v}-1}\left(\sum_{j=1}^{3} \frac{\partial \varphi^{\mathrm{e}}}{\partial \varepsilon_{j}^{\mathrm{e}}} \frac{1}{2 c_{j}^{\mathrm{pr}}} \mathbf{E}_{j}^{\mathrm{pr}}\right) \mathbf{F}_{n}^{\mathrm{v}-\mathrm{T}}
\end{aligned}
$$

The choice between expressions (62) or (63) is just a matter of implementation convenience. Nevertheless, in this work (63) will be preferred to compute second derivatives due to the fact that $\mathbf{F}_{n}^{\mathrm{v}}$ does not depend on $\hat{\mathbf{C}}_{n+1}$.

Three additional remarks are useful to enhance the characteristics of the present framework:

i. Potentials $\varphi$ and $\phi$ from the Kelvin branch are uncoupled and out of the minimization operations. Thus, their choice does not affect the elastic/viscous decomposition on the Maxwell branch.

ii. The four non-linear scalar equations (56), (57) are simple enough to allow any choice of potentials $\varphi^{\mathrm{e}}$ and $\psi$. Thus, no restriction is made on the size of perturbations away from the thermodynamic equilibrium up to this point [10]. Moreover, convenient expressions of $\varphi^{\mathrm{e}}$ and $\psi$ may simplify system (56), (57) even more, as is shown in the next section.

iii. A simple extension to this model can be obtained by considering a set of $P$ Maxwell branches, as seen in Figure 1. In this case, the incremental potential (45) is

$$
\begin{aligned}
\Psi\left(\mathbf{C}_{n+1} ; \mathscr{E}_{n}\right)= & \Delta \varphi\left(\hat{\mathbf{C}}_{n+1}\right)+\Delta t \phi\left(\frac{\Delta q_{j}}{\Delta t}\right)+\Delta U\left(\theta_{n+1}\right) \\
& +\sum_{k=1}^{P} \min _{\mathbf{M}_{j k}^{\mathrm{v}}, \Delta q_{j k}^{\mathrm{v}}}\left\{\Delta \varphi_{k}^{\mathrm{e}}\left(\hat{\mathbf{C}}_{k n+1}^{\mathrm{e}}\right)+\Delta t \psi_{k}\left(\frac{\Delta q_{j k}^{\mathrm{v}}}{\Delta t}\right)\right\}
\end{aligned}
$$

which means that the minimization should be performed for each $k$-module, thus obtaining the corresponding pair $\Delta q_{j k}^{\mathrm{v}}, \mathbf{M}_{j k}$.

A final aspect concerns the computation of the incremental dissipation, which is a fundamental point for further extensions to thermomechanical models. Dissipated energy in the present model is associated with the Kelvin and Maxwell contributions from the corresponding dissipation potentials $\phi$ and $\psi$. The increment of dissipated energy $\Delta D$ at each time step is computed as 
the time integration of the product of conjugated forces times the corresponding stretchings. Taking advantage of the isotropic properties of the potentials,

$$
\begin{aligned}
\Delta D & =\Delta D^{\mathrm{K}}+\Delta D^{\mathrm{M}} \\
\Delta D^{\mathrm{K}} & =\left.\int_{t_{n}}^{t_{n+1}} \sum_{j=1}^{3} \frac{\partial \phi}{\partial d_{j}} d_{j} \mathrm{~d} t \simeq \sum_{j=1}^{3} \frac{\partial \phi}{\partial d_{j}}\right|_{\bar{t}} d_{j} \Delta t=\left.\sum_{j=1}^{3} \frac{\partial \phi}{\partial d_{j}}\right|_{\bar{t}} \Delta q_{j} \\
\Delta D^{\mathrm{M}} & =\left.\int_{t_{n}}^{t_{n+1}} \sum_{j=1}^{3} \frac{\partial \psi}{\partial d_{j}^{\mathrm{v}}} d_{j}^{\mathrm{v}} \mathrm{d} t \simeq \sum_{j=1}^{3} \frac{\partial \psi}{\partial d_{j}^{\mathrm{v}}}\right|_{\bar{t}} d_{j}^{\mathrm{v}} \Delta t=\left.\sum_{j=1}^{3} \frac{\partial \psi}{\partial d_{j}^{\mathrm{v}}}\right|_{\bar{t}} \Delta q_{j}^{\mathrm{v}}
\end{aligned}
$$

where $t_{n} \leqslant \bar{t} \leqslant t_{n+1}$ and $\Delta D^{\mathrm{K}}, \Delta D^{\mathrm{M}}$ are the respective contributions of the Kelvin and Maxwell branches. Due to the assumed properties of $\phi$ and $\psi$, dissipation is clearly non-negative.

\subsection{Material tensors}

An important aspect from the numerical implementation point of view is the determination of the tangent matrix, consistent with the constitutive incremental update algorithm. The contribution to the tangent matrix from geometric terms is common to any hyperelastic model based on the variational balance equations (5)-(7). Thus, we focus here on the expression of the second derivative of the present incremental material update. We will use here the notation $\mathrm{d}(\cdot) / \mathrm{d} \hat{\mathbf{C}}_{n+1}$ as the total derivative of the argument with respect to $\hat{\mathbf{C}}_{n+1}$. We define thus the tensor $\mathscr{C}$ :

$$
\begin{aligned}
\mathscr{C} & =\frac{\mathrm{d}}{\mathrm{d} \hat{\mathbf{C}}_{n+1}}\left(\frac{\partial \Psi}{\partial \hat{\mathbf{C}}_{n+1}}\right)=\frac{\mathrm{d}^{2} \varphi}{\left(\mathrm{d} \hat{\mathbf{C}}_{n+1}\right)^{2}}+\frac{\mathrm{d}^{2} \phi}{\left(\mathrm{d} \hat{\mathbf{C}}_{n+1}\right)^{2}}+\frac{\mathrm{d}}{\mathrm{d} \hat{\mathbf{C}}_{n+1}}\left(\frac{\partial \varphi^{\mathrm{e}}}{\partial \hat{\mathbf{C}}_{n+1}}\right) \\
& =\mathscr{C}^{\varphi}+\mathscr{C}^{\phi}+\mathscr{C}^{\varphi^{\mathrm{e}}}
\end{aligned}
$$

The first two members are quite easy to compute. Indeed, since $\varphi$ is an isotropic function of $\hat{\mathbf{C}}_{n+1}$, its second derivative is easily computed in the spectral co-ordinate system (see, for example, Reference [17]). The second term needs a preliminary pull-back operation by tensor $\hat{\mathbf{F}}_{n}^{-1}$. In order to facilitate notation, we call $\mathbf{f}^{n}=\hat{\mathbf{F}}_{n}^{-1}$ and the index $n+1$ is dropped. Using the relation $\Delta \hat{\mathbf{C}}=\hat{\mathbf{F}}_{n}^{-\mathrm{T}} \hat{\mathbf{C}}_{n+1} \hat{\mathbf{F}}_{n}^{-1}=\mathbf{f}^{n \mathrm{~T}} \hat{\mathbf{C}} \mathbf{f}^{n}$, we obtain the following symmetric fourthorder tensor (see Appendix):

$$
\mathscr{C}_{i j k l}^{\phi}=\frac{\partial}{\partial \hat{\mathbf{C}}_{k l}}\left(\frac{\partial \phi}{\partial \hat{\mathbf{C}}_{i j}}\right)=\sum_{m, t, p, q=1}^{3} \mathbf{f}_{i m}^{n} \mathbf{f}_{j t}^{n} \frac{\partial \phi}{\partial \Delta \hat{\mathbf{C}}_{m t} \partial \Delta \hat{\mathbf{C}}_{p q}} \mathbf{f}_{k p}^{n} \mathbf{f}_{l q}^{n}=\mathscr{C}_{k l i j}^{\phi}=\mathscr{C}_{j i k l}^{\phi}
$$

Since $\phi$ is an isotropic function of $\Delta \hat{\mathbf{C}}$, its second derivative is easily obtained using spectral co-ordinates in a way analogous to the one for $\varphi$. 
The third term is treated in an equivalent fashion. Considering $\hat{\mathbf{C}}^{\mathrm{pr}}=\hat{\mathbf{F}}_{n}^{\mathrm{v}-\mathrm{T}} \hat{\mathbf{C}}_{n+1} \hat{\mathbf{F}}_{n}^{\mathrm{v}-1}$, calling $\mathbf{f}^{\mathrm{v} n}=\hat{\mathbf{F}}_{n}^{\mathrm{v}-1}$ and dropping index $n+1$, we have

$$
\mathscr{C}_{i j k l}^{\varphi^{\mathrm{e}}}=\frac{\mathrm{d}}{\mathrm{d} \hat{\mathbf{C}}_{k l}}\left(\frac{\partial \varphi^{\mathrm{e}}}{\partial \hat{\mathbf{C}}_{i j}}\right)=\sum_{m, t, p, q=1}^{3} \mathbf{f}_{i m}^{\mathrm{v} n} \mathbf{f}_{j t}^{\mathrm{v} n} \frac{\mathrm{d}}{\mathrm{d} \hat{\mathbf{C}}_{p q}^{\mathrm{pr}}}\left(\frac{\partial \varphi^{\mathrm{e}}}{\partial \hat{\mathbf{C}}_{m t}^{\mathrm{p}}}\right) \mathbf{f}_{k p}^{\mathrm{v} n} \mathbf{f}_{l q}^{\mathrm{v} n}=\mathscr{C}_{k l i j}^{\varphi^{\mathrm{e}}}=\mathscr{C}_{j i k l}^{\varphi^{\mathrm{e}}}
$$

The critical point is the obtainment of the derivatives of $\varphi^{\mathrm{e}}$ with respect to $\hat{\mathbf{C}}^{\mathrm{pr}}=c_{j}^{\mathrm{pr}} \mathbf{E}_{j}^{\mathrm{pr}}$. In spectral co-ordinates this requires the computation of the following functions (see Appendix for details):

$$
\begin{aligned}
y_{i} & =\frac{\partial \varphi^{\mathrm{e}}}{\partial c_{i}^{\mathrm{pr}}}=\frac{\partial \varphi^{\mathrm{e}}}{\partial \varepsilon_{i}^{\mathrm{e}}} \frac{1}{2 c_{i}^{\mathrm{pr}}} \\
y_{i, j} & =\frac{\mathrm{d}}{\mathrm{d} c_{j}^{\mathrm{pr}}}\left(\frac{\partial \varphi^{\mathrm{e}}}{\partial \varepsilon_{i}^{\mathrm{e}}} \frac{1}{2 c_{i}^{\mathrm{pr}}}\right)=\frac{\partial^{2} \varphi^{\mathrm{e}}}{\partial \varepsilon_{i}^{\mathrm{e}} \partial \varepsilon_{i}^{\mathrm{e}}} \frac{\mathrm{d} \varepsilon_{i}^{\mathrm{e}}}{\mathrm{d}_{j}^{\mathrm{pr}}} \frac{1}{4 c_{i}^{\mathrm{pr}} c_{j}^{\mathrm{pr}}}-\frac{\partial \varphi^{\mathrm{e}}}{\partial \varepsilon_{i}^{\mathrm{e}}} \frac{1}{2\left(c_{i}^{\mathrm{pr}}\right)^{2}}
\end{aligned}
$$

The terms $\partial \varphi^{\mathrm{e}} / \partial \varepsilon_{k}^{\mathrm{e}}$ and $\partial^{2} \varphi^{\mathrm{e}} / \partial \varepsilon_{k}^{\mathrm{e}} \partial \varepsilon_{l}^{\mathrm{e}}$ are straightforward. On the other hand, the relation $\varepsilon_{k}^{\mathrm{e}}\left(\varepsilon_{1}^{\mathrm{pr}}, \varepsilon_{2}^{\mathrm{pr}}, \varepsilon_{3}^{\mathrm{pr}}\right)$ is defined by the derivation of the non-linear system (58), (59) (see Appendix):

$$
\begin{aligned}
& \frac{\partial r_{i}}{\partial \varepsilon_{j}^{\mathrm{pr}}}=K_{i i} \frac{\mathrm{d} \varepsilon_{i}^{\mathrm{e}}}{\mathrm{d} \varepsilon_{j}^{\mathrm{pr}}}-\left(\psi_{, i i} \frac{\delta_{i j}}{\Delta t}-\frac{\mathrm{d} \lambda}{\mathrm{d} \varepsilon_{j}^{\mathrm{pr}}}\right)=0, \quad i=1,2,3 \\
& \frac{\partial r_{4}}{\partial \varepsilon_{j}^{\mathrm{pr}}}=\frac{\mathrm{d} \varepsilon_{1}^{\mathrm{e}}}{\mathrm{d} \varepsilon_{j}^{\mathrm{pr}}}+\frac{\mathrm{d} \varepsilon_{2}^{\mathrm{e}}}{\mathrm{d} \varepsilon_{j}^{\mathrm{pr}}}+\frac{\mathrm{d} \varepsilon_{3}^{\mathrm{e}}}{\mathrm{d} \varepsilon_{j}^{\mathrm{pr}}}=0
\end{aligned}
$$

where we use the notation

$$
\psi_{, i}=\frac{\partial \psi}{\partial d_{i}^{\mathrm{v}}}, \quad \varphi_{, i}=\frac{\partial \varphi}{\partial \varepsilon_{i}^{\mathrm{e}}}, \quad K_{i i}=\varphi_{, i i}+\frac{1}{\Delta t} \psi_{, i i}
$$

Isolating $\mathrm{d} \varepsilon_{i}^{\mathrm{e}} / \mathrm{d} \varepsilon_{j}^{\mathrm{pr}}$ from (70), substituting in (71), and returning back to (70) we obtain the explicit expression

$$
\frac{\mathrm{d} \varepsilon_{i}^{\mathrm{e}}}{\mathrm{d} \varepsilon_{j}^{\mathrm{pr}}}=\frac{\psi_{, i i}}{\Delta t K_{i i}} \delta_{i j}-\frac{\psi_{, j j}}{\Delta t K_{j j} K_{i i}}\left(\sum_{s=1}^{3} \frac{1}{K_{s s}}\right)^{-1}
$$

\subsection{Hencky-based model}

In this section we analyse the case when potentials are based on quadratic forms of logarithmic strain tensors (Hencky-type potentials $[16,17]$ )

$$
\begin{gathered}
\varphi=\mu \sum_{j=1}^{3}\left(\varepsilon_{j}\right)^{2}, \quad \phi=\eta \sum_{j=1}^{3}\left(d_{j}\right)^{2} \\
\varphi^{\mathrm{e}}=\mu^{\mathrm{e}} \sum_{j=1}^{3}\left(\varepsilon_{j}^{\mathrm{e}}\right)^{2}, \quad \psi=\eta^{\mathrm{v}} \sum_{j=1}^{3}\left(d_{j}^{\mathrm{v}}\right)^{2}
\end{gathered}
$$


From (55) we have that $\varepsilon_{j}^{\mathrm{e}}=\varepsilon_{j}^{\mathrm{pr}}-\Delta t d_{j}^{\mathrm{v}}=\varepsilon_{j}^{\mathrm{pr}}-\Delta q_{j}^{\mathrm{v}}$. Using (75), the optimality conditions (56), (57) take the particular form

$$
\begin{gathered}
-2 \mu^{\mathrm{e}}\left(\varepsilon_{j}^{\mathrm{pr}}-\Delta q_{j}^{\mathrm{v}}\right)+2 \eta^{\mathrm{v}} \frac{\Delta q_{j}^{\mathrm{v}}}{\Delta t}+\lambda=0, \quad j=1,2,3 \\
\Delta q_{j}^{\mathrm{v}}+\Delta q_{j}^{\mathrm{v}}+\Delta q_{j}^{\mathrm{v}}=0
\end{gathered}
$$

It is easy to see that, summing up all three linear equations (76), the constraint (77) and the fact that $\varepsilon_{1}^{\mathrm{pr}}+\varepsilon_{2}^{\mathrm{pr}}+\varepsilon_{3}^{\mathrm{pr}}=0$, provides $\lambda=0$. This fact decouples the system and we finally have

$$
\Delta q_{j}^{\mathrm{v}}=\frac{\mu^{\mathrm{e}}}{\frac{\eta^{\mathrm{v}}}{\Delta t}+\mu^{\mathrm{e}}} \varepsilon_{j}^{\mathrm{pr}}
$$

for each principal direction $j$, which is the classical expression of the corresponding infinitesimal linear viscoelastic model. The particular derivatives $\partial \varphi / \partial \varepsilon_{j}, \partial \phi / \partial d_{j}, \partial \varphi^{\mathrm{e}} / \partial \varepsilon_{j}^{\mathrm{e}}, \partial \psi / \partial d_{j}^{\mathrm{v}}$ and higher orders needed to substitute into (60), (63) and (73) are easily obtained from the potentials definition.

\subsection{Ogden-based model}

In the previous case, the quadratic function of the logarithmic strains is particularly convenient to obtain the simple uncoupled linear expression (78) for the minimizing argument $\Delta q_{j}^{\mathrm{v}}$. In spite of this advantage, it is well known that this type of hyperelastic potentials do not fit very well the behaviour of rubber-like materials, which are the ones that most commonly exhibit viscoelastic properties. For that, a more adequate choice may be the Ogden model [16, 17] which also has the property of generalizing other models like neo-Hookean and Mooney-Rivlin.

Different combinations are possible. A first attempt may be to associate the main spring of the Kelvin branch with an Ogden model, leaving the Maxwell branch with a Hencky-type definition, as was done in the previous section. This option seems to provide small viscous stress contributions for large strain ranges, when compared with the stress obtained by the Ogden model in the main spring. This fact will be more easily understood with a corresponding numerical example in the next section. Another possibility is defining both springs of the Ogden type. Consider then the following potentials (the same symbol $\alpha_{p}$ was used in all expressions for the purpose of simplicity, but it can be different for each function):

$$
\begin{aligned}
& \varphi=\sum_{j=1}^{3} \sum_{p=1}^{N} \frac{\mu_{p}}{\alpha_{p}}\left(\left[\exp \left(\varepsilon_{j}\right)\right]^{\alpha_{p}}-1\right) \\
& \phi=\sum_{j=1}^{3} \sum_{p=1}^{N} \frac{\eta_{p}}{\alpha_{p}}\left(\left[\exp \left(d_{j}\right)\right]^{\alpha_{p}}-1\right)
\end{aligned}
$$




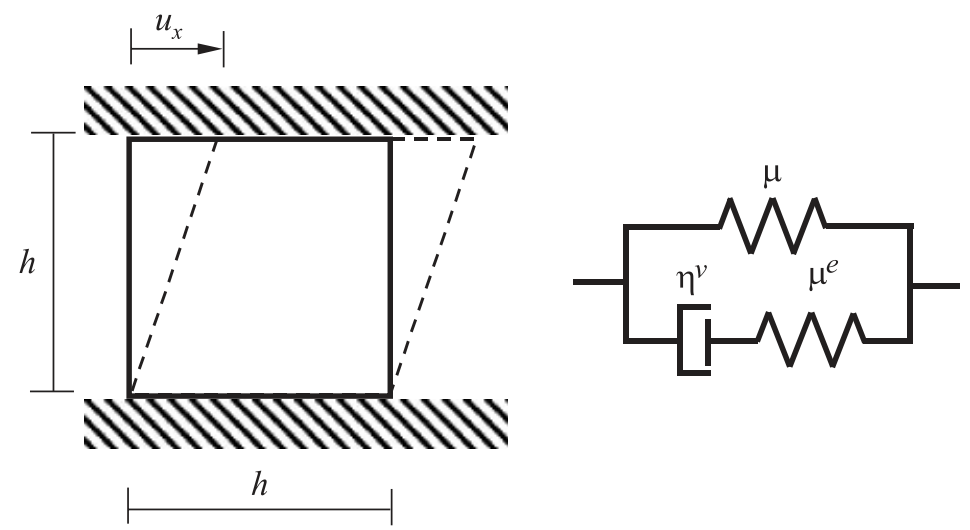

Figure 3. Cyclic shear test.

$$
\begin{aligned}
\varphi^{\mathrm{e}} & =\sum_{j=1}^{3} \sum_{p=1}^{N} \frac{\mu_{p}^{\mathrm{e}}}{\alpha_{p}}\left(\left[\exp \left(\varepsilon_{j}^{\mathrm{e}}\right)\right]^{\alpha_{p}}-1\right) \\
\psi & =\sum_{j=1}^{3} \sum_{p=1}^{N} \frac{\eta_{p}^{\mathrm{v}}}{\alpha_{p}}\left(\left[\exp \left(d_{j}^{\mathrm{v}}\right)\right]^{\alpha_{p}}-1\right)
\end{aligned}
$$

Substituting (81) and (82) in (56), (57) we obtain the non-linear set

$$
\begin{gathered}
\sum_{p=1}^{N} \eta_{p}^{\mathrm{e}} \exp \left(\alpha_{p} d_{j}^{\mathrm{v}}\right)-\sum_{p=1}^{N} \mu_{p}^{\mathrm{e}} \exp \left(\alpha_{p} \varepsilon_{j}^{\mathrm{e}}\right)+\lambda=0, \quad j=1,2,3 \\
d_{1}^{\mathrm{v}}+d_{2}^{\mathrm{v}}+d_{3}^{\mathrm{v}}=0
\end{gathered}
$$

Variables $d_{j}^{\mathrm{v}}, \lambda$ can be solved by, for example, the Newton procedure (see Appendix). Again, particular derivatives $\partial \varphi / \partial \varepsilon_{j}, \partial \phi / \partial d_{j}, \partial \varphi^{\mathrm{e}} / \partial \varepsilon_{j}^{\mathrm{e}}, \partial \psi / \partial d_{j}^{\mathrm{v}}$ and higher orders needed to substitute in (60), (63) and (73) are easily obtained from the potentials definition.

\section{NUMERICAL EXAMPLES}

This section shows some numerical examples with the aim of discussing performance and particular characteristics of the chosen models (potentials) within the present formulation. Finite element calculations were performed using the $\mathrm{C}++$ version of the finite element program METAFOR [20].

\subsection{Cyclic shear test}

Consider a pure shear test of a single 3D element (Figure 3). This example was extracted from Reference [10] and its goal is to illustrate some characteristics of the present approach. 
Table I. Material parameters for cyclic shear test.

\begin{tabular}{lrrrc}
\hline Potential & \multicolumn{3}{c}{ Ogden } & Hencky \\
\hline$\varphi\left(\mu_{i}\right)$ & 20 & -7 & 1.5 & $\mu=30.25=\sum_{i} \frac{1}{2} \mu_{i} \alpha_{i}$ \\
$\varphi^{\mathrm{e}}\left(\mu_{i}^{\mathrm{e}}\right)$ & 51.4 & -18 & 3.86 & $\mu^{\mathrm{e}}=77.77=\sum_{i} \frac{1}{2} \mu_{i}^{\mathrm{e}} \alpha_{i}$ \\
$\psi\left(\eta_{i}\right)$ & 899.5 & -315 & 67.55 & $\eta^{\mathrm{v}}=1361=\sum_{i} \frac{1}{2} \eta_{i}^{\mathrm{v}} \alpha_{i}$ \\
\hline
\end{tabular}

Table II. Material model combinations.

\begin{tabular}{lccc}
\hline Case & $\varphi$ & $\varphi^{\mathrm{e}}$ & $\psi$ \\
\hline 1 & Hencky & Hencky & Hencky \\
2 & Ogden & Hencky & Hencky \\
3 & Ogden & Ogden & Hencky \\
4 & Ogden & Ogden & Ogden \\
\hline
\end{tabular}

Moreover, if appropriate potentials are chosen, we show that results equivalent to those of Reference [10] are obtained. The rheological model chosen for this example corresponds to the one of Figure 3 (potentials $\phi=0$ ).

This example consists of a pure shear test of a single 3D element (Figure 3). Material parameters and load characteristics were taken from the same example in Reference [10], in order to perform numerical comparisons of results when possible. The rheological model chosen for this example corresponds to the one of Figure 3 (potentials $\phi=0$ ).

The lateral displacement follows a sinusoidal law $u_{x}=U_{x} \sin w t$, where $w=0.3 \mathrm{~s}^{-1}$. The quotient $u_{x} / h$ is numerically equal to the shear component $\mathbf{C}_{x y}$ (or $\mathbf{B}_{x y}$ ) in the Cartesian coordinate system. The material was assumed to be almost incompressible through a convenient penalization value of $K$.

In addition, Ogden and Hencky models were used for $\varphi, \varphi^{\mathrm{e}}$ and $\psi$. For Ogden models we used $N=3$, i.e. $p=1,2,3$. Also, to reduce the number of free parameters, we assume $\mu_{p}^{\mathrm{e}}=2.57 \mu_{p}$ and viscous coefficients $\eta_{p}^{\mathrm{v}}$ such that $\tau=\eta_{\mathrm{p}}^{\mathrm{v}} / \mu_{p}^{\mathrm{e}}=17.5$. Moreover, the same Ogden exponents $\alpha_{p}$ were used for all potentials: $\alpha_{1}=1.8 ; \alpha_{2}=-2 ; \alpha_{3}=7$ (see Table I).

For the Hencky cases, the value $\mu=\sum_{p} \frac{1}{2} \mu_{p} \alpha_{p}$ was used, which is the consistent equivalent parameter for small strains. The four combinations shown in Table II were tested.

The following graphics show the behaviour of Cauchy stresses $\sigma_{x y}$ as a function of shear strain $\mathbf{C}_{x y}$ in time, for different shear amplitudes: 0.01, 1,2 and 5. The total time is such that 3(three) load cycles are completed. In the case of small strains shown in Figure 4, all models give identical results, as expected. For larger amplitudes, quite different results are found. The results of cases 2-4 shown in Figures 5-10, present a close correlation of maximum values of stress with the corresponding results found in Reference [10]. However, hysteresis loops of case 2, are clearly 'thinner' as the deformation grows along the cycle. This behaviour is in agreement with the fact that the Hencky model used in the Maxwell branch provides a contribution in stress much lower than a corresponding Ogden model for large strains. Cases 3 


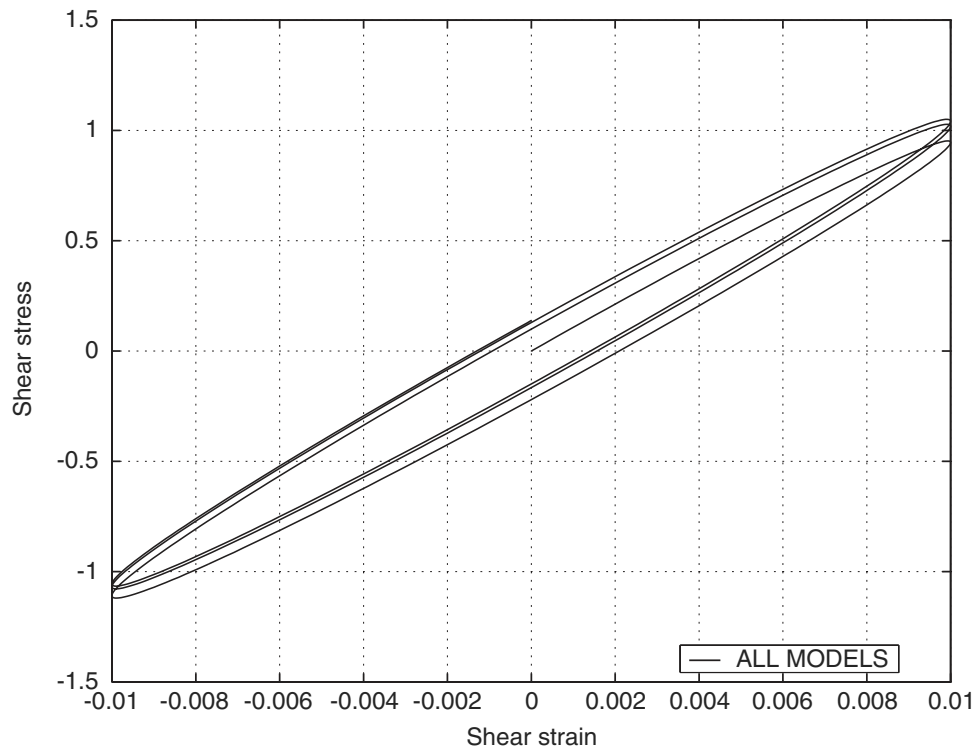

Figure 4. Cyclic shear test. Shear amplitude: 0.01 .

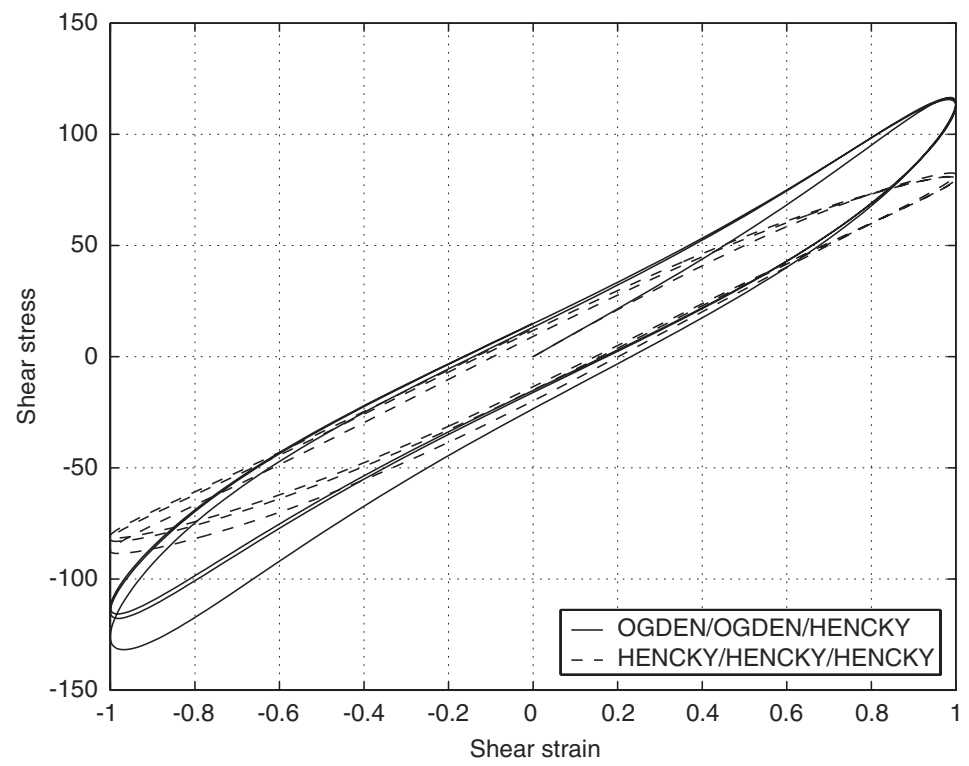

Figure 5. Cyclic shear test. Shear amplitude: 1.

and 4 graphically fit the non-linear case of Reference [10]. Moreover, they provide almost the same results in this example, where a proportionality $\tau$ among elastic and viscous parameters was used. 


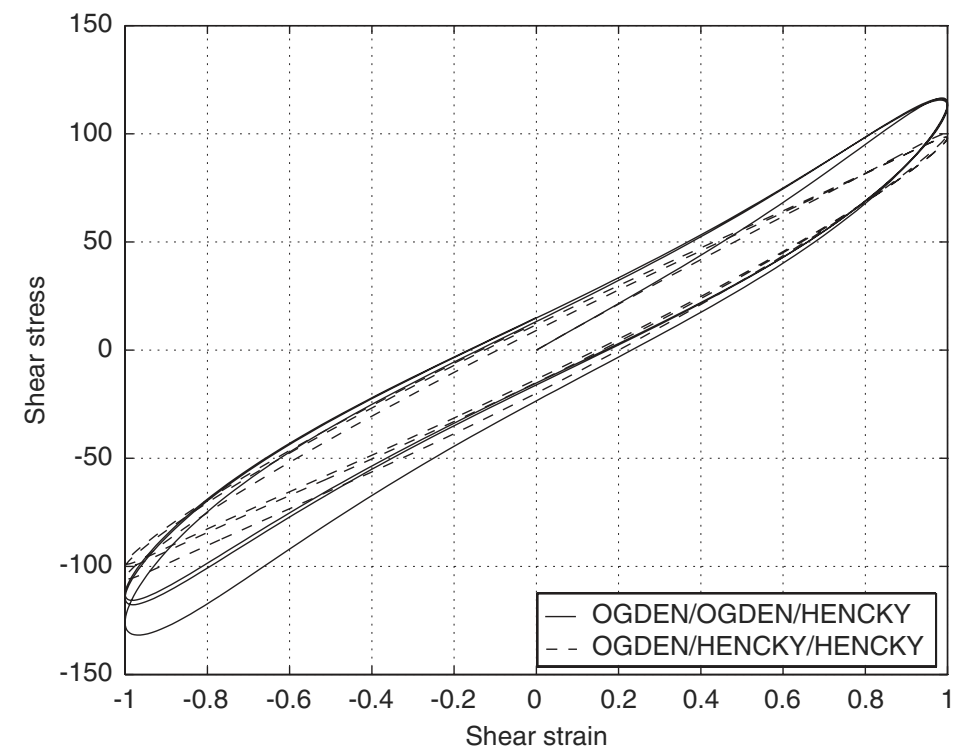

Figure 6. Cyclic shear test. Shear amplitude: 1.

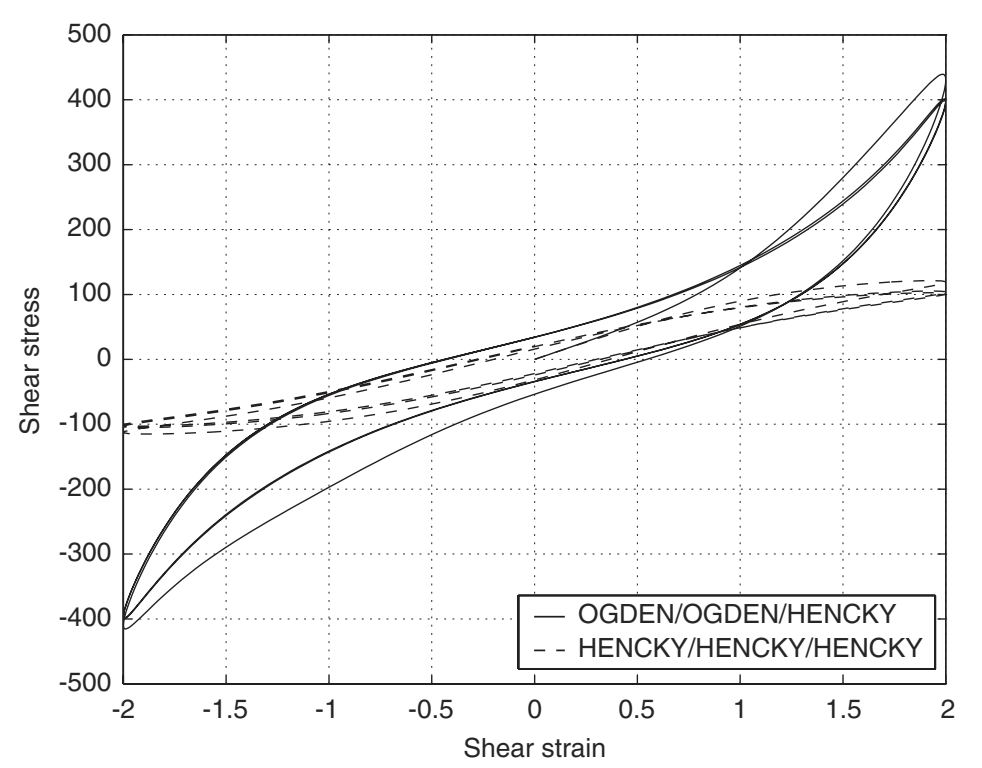

Figure 7. Cyclic shear test. Shear amplitude: 2.

The viscous potential modifies the minimization condition (56), (57) and, thus, the amount of viscous deformation. In order to put in evidence its influence we ran two more cases in which the main spring is eliminated and the Maxwell spring keeps its previous values. The potential $\psi$ changes from the Hencky model (with the same viscosity $\eta^{v}$ already used) to the Ogden model, 


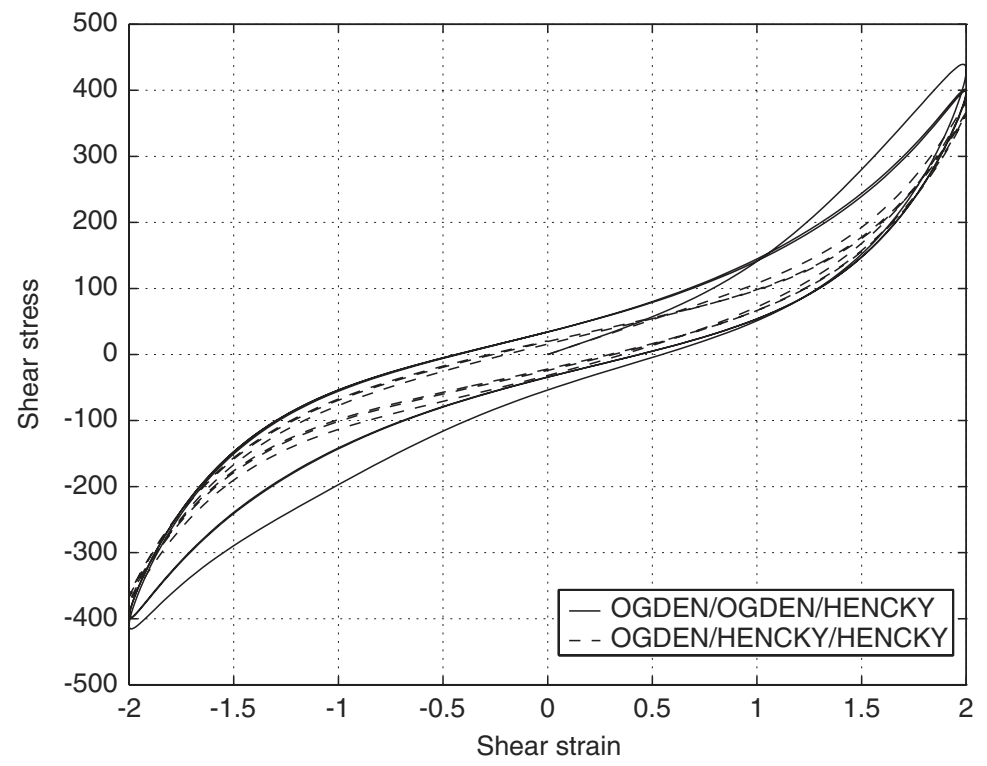

Figure 8. Cyclic shear test. Shear amplitude: 2 .

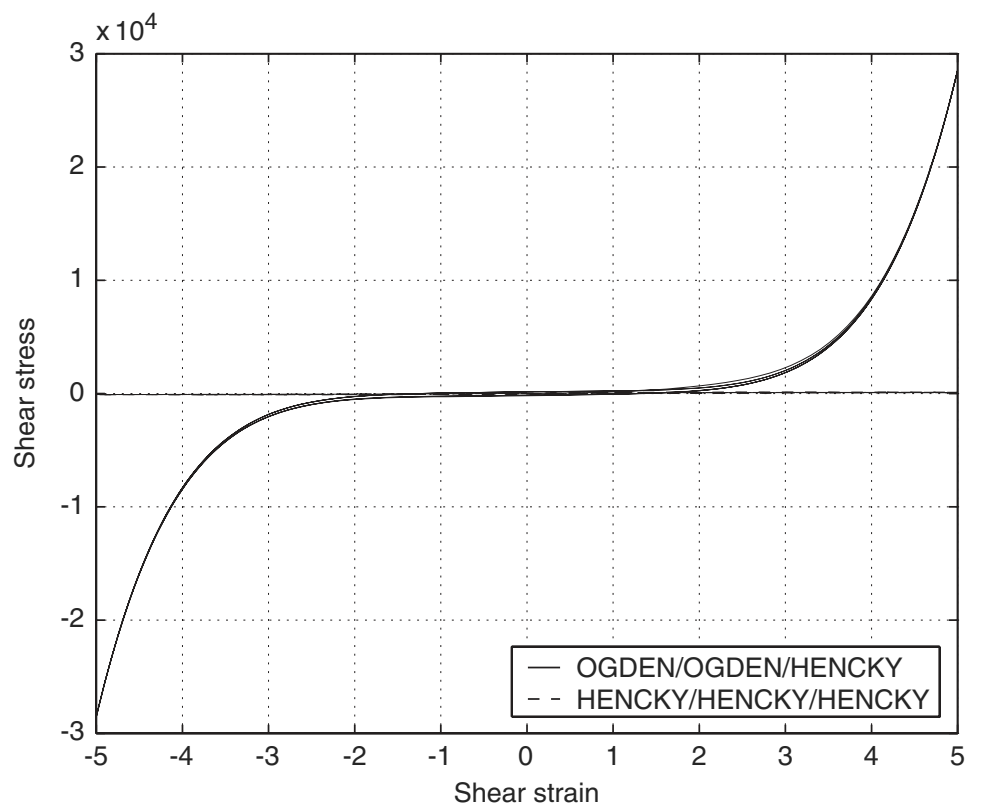

Figure 9. Cyclic shear test. Shear amplitude: 5.

using parameters with the same equivalent viscosity $\eta^{\mathrm{v}}=\sum_{p} \frac{1}{2} \eta_{p}^{\mathrm{v}} \alpha_{p}$ (see Table III). Again, in the small strain case, all models provide the same behaviour (Figure 11). On the other side, finite deformations clearly exhibit differences on the hysteresis loop, as shown in Figures 12 and 13 . 


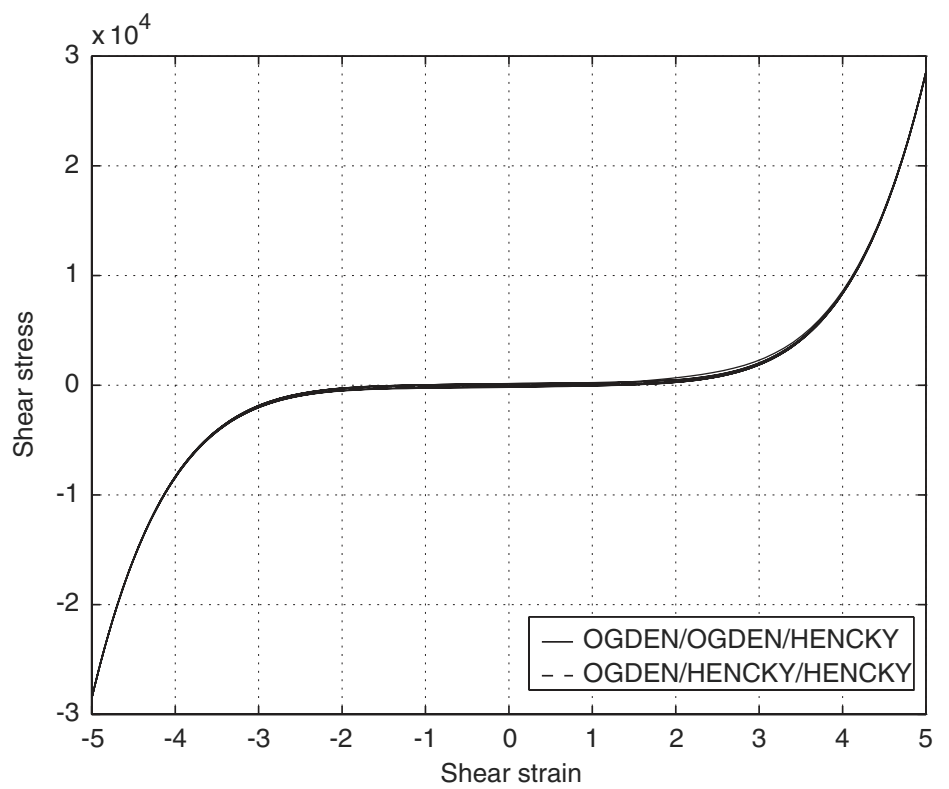

Figure 10. Cyclic shear test. Shear amplitude: 5 .

Table III. Material parameters for potential $\psi$.

\begin{tabular}{lcc}
\hline Ogden (a) & Ogden (b) & Hencky \\
\hline$\eta_{1}^{\mathrm{v}}=272.2$ & $\eta_{1}^{\mathrm{v}}=136.1$ & $\eta^{\mathrm{v}}=1361=\frac{1}{2} \eta_{1}^{\mathrm{v}} \alpha_{1}$ \\
$\alpha_{1}=10$ & $\alpha_{1}=20$ & \\
\hline
\end{tabular}

\subsection{Pinched cylinder}

The goal of the next examples is to show the applicability of the present model to typical large-scale FEM computations. Figure 14(a) shows a thick viscoelastic cylinder and a rigid hemispherical tool following the path shown in Figure 14(b): first, it pinches the cylinder down to a cursor-end position where it remains during a relaxing period. Finally, it is removed at high velocity, which produces different final piece shapes and different times of separation, depending on the initial tool velocity defined by the time $t$ (see Figure 14(b)). Five cases are tested with $t=0.25,0.5,1,2$ and $3 \mathrm{~s}$. This example is inspired from a similar one found in Reference [3].

Cylinder dimensions are $R_{\mathrm{i}}=12 \mathrm{~mm}, R_{\mathrm{e}}=16 \mathrm{~mm}$ and $L=20 \mathrm{~mm}$. The tool has a radius of $2.5 \mathrm{~mm}$ and its displacement is equal to $R_{\mathrm{i}}$. An Ogden model with the same parameters as in the previous example was used for both spring potentials $\varphi$ and $\varphi^{\mathrm{e}}: \mu_{1}=2.758 ; \mu_{2}=-1.725$; $\mu_{3}=0.704(\mathrm{MPa})$ and $\alpha_{1}=1.33 ; \alpha_{2}=-3.05 ; \alpha_{3}=3.89$. The viscous potential $\psi$ is of the 


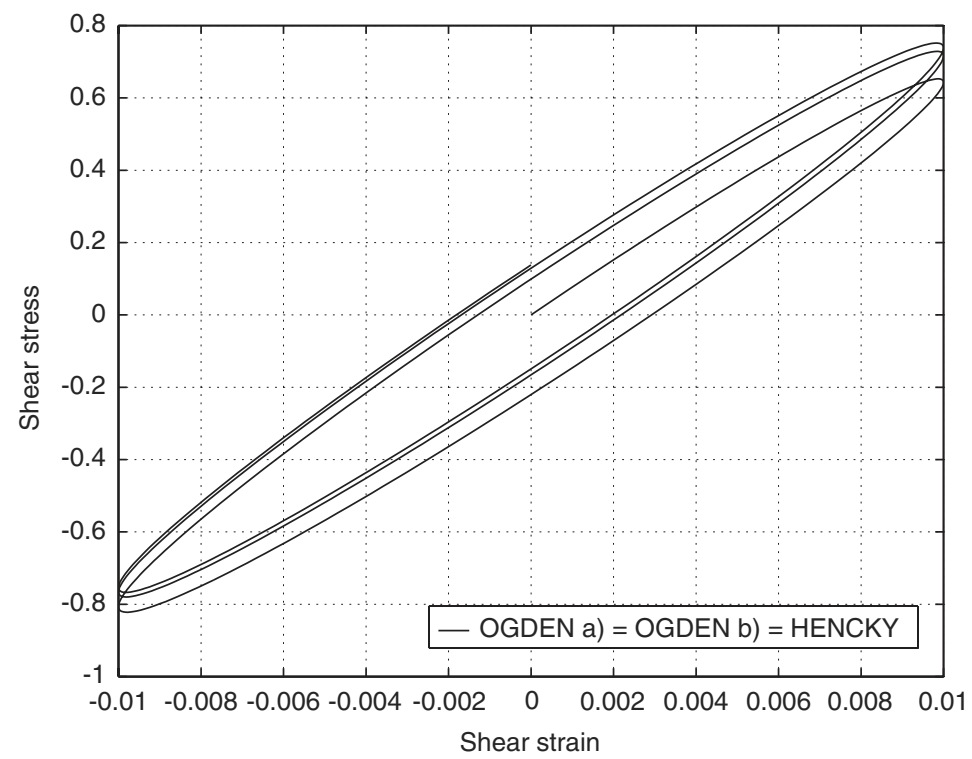

Figure 11. Cyclic shear test. Influence of viscous potential. Shear amplitude: 0.01 .

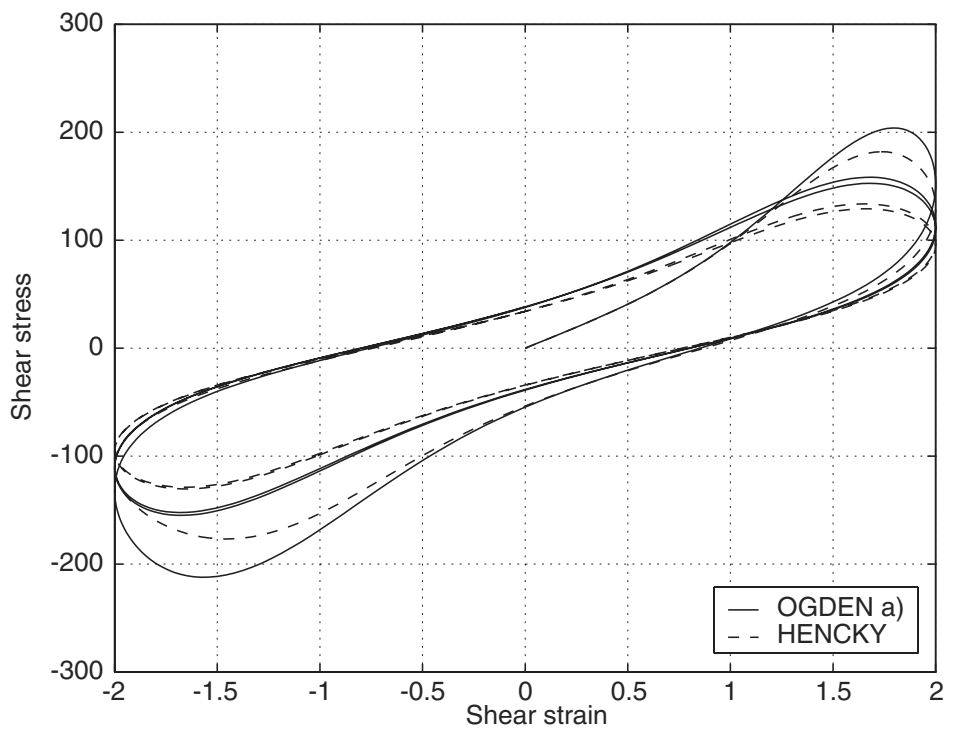

Figure 12. Cyclic shear test. Influence of viscous potential. Shear amplitude: 2.

Hencky type, with $\eta^{\mathrm{v}}=1.1394(\tau=2 \mathrm{~s})$. Potential $\phi$ is null. Lateral faces of the cylinder are free and symmetry of the model was taken into account for the analysis.

Figure 15(a) shows the deformed configuration for the maximum tool displacement, while 15(b) illustrates the final configuration after tool removal. The history of the Cauchy 


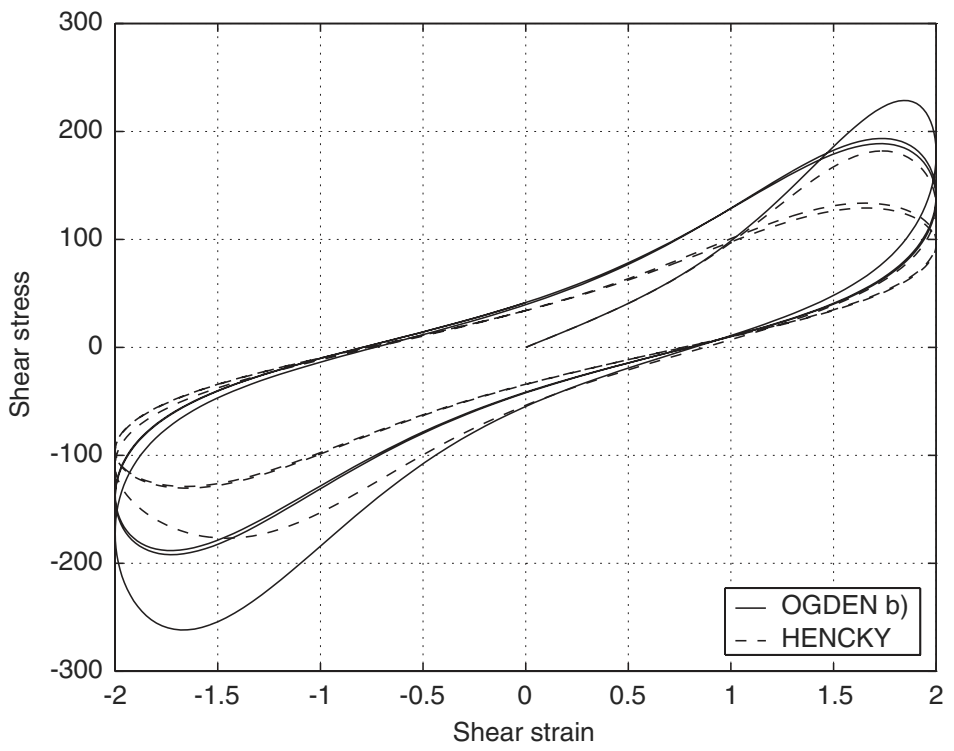

Figure 13. Cyclic shear test. Influence of viscous potential. Shear amplitude: 2.
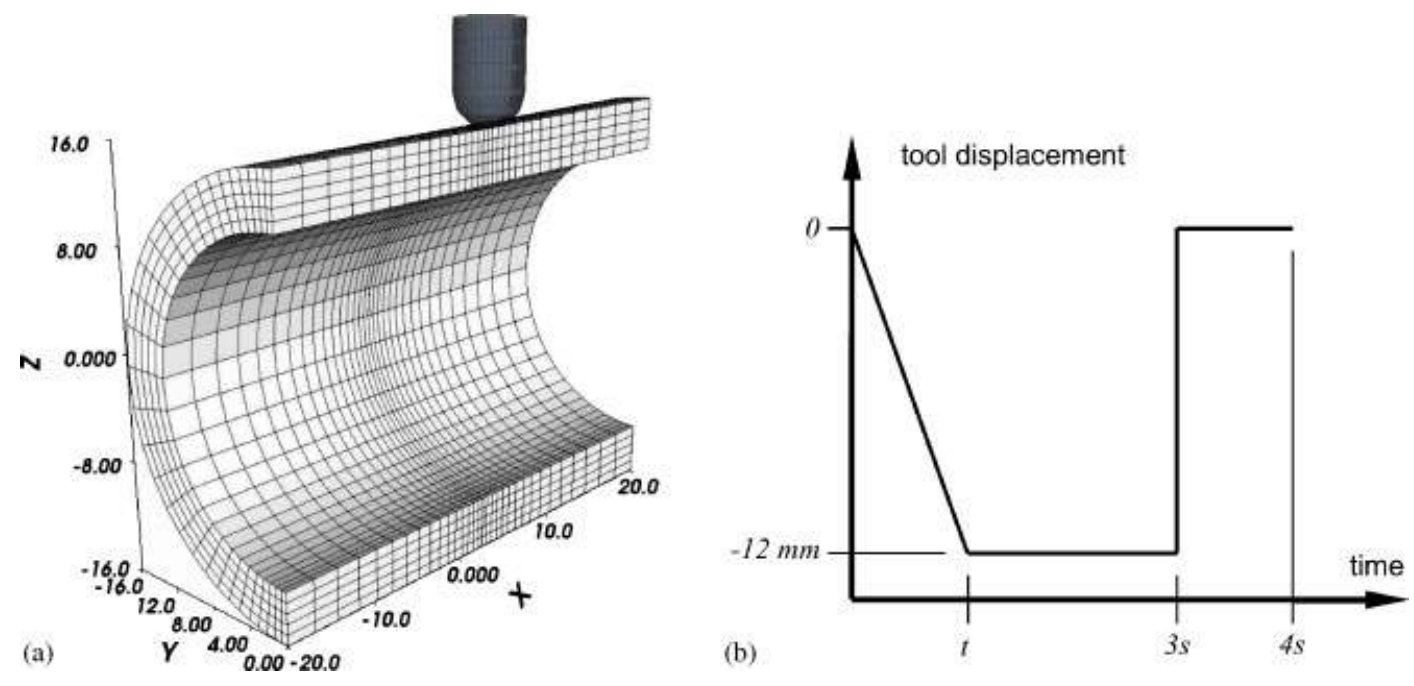

Figure 14. (a) Pinched cylinder; and (b) tool path.

stress $\sigma_{x}$ and displacement of the point located on the cylinder interior surface just below the punch tip is plotted in Figure 16. The history of the former point displacement is shown in Figure 17. As expected, the faster the punch is applied, the higher the stresses that are developed and the higher the dissipated energy at the tool removal time $t=3 \mathrm{~s}$. Consequently, 


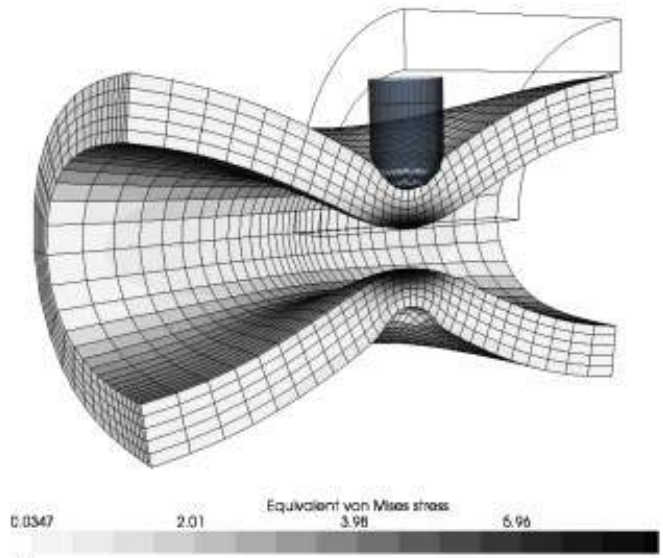

(a)

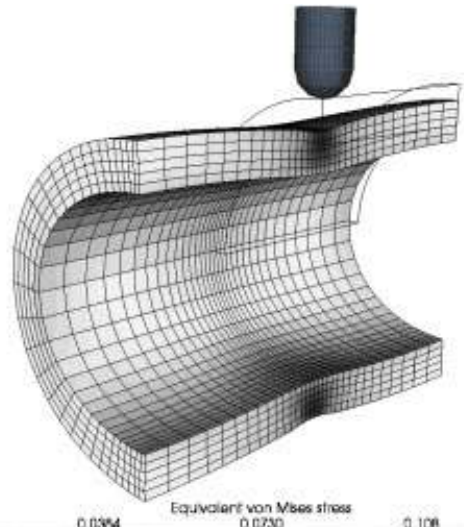

(b)

Figure 15. Pinched cylinder: (a) intermediate; and (b) final configurations.

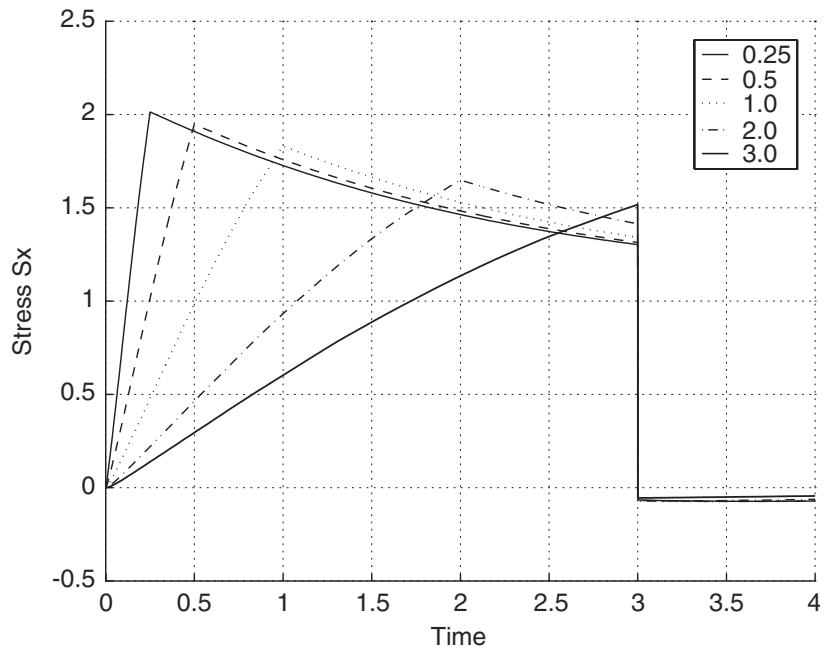

Figure 16. Cauchy stress $\sigma_{x}$ versus time.

owing to a higher relaxation in the system, we see a faster separation from the tool during its backward motion.

\subsection{Viscoelastic support}

This example simulates a viscoelastic cylindrical support clamped on its exterior surface and with a rigid edge connected to a vibrating device (see Figure 18).

The harmonic vibration $u=U \sin w t$, with $w=10 \mathrm{~s}^{-1}$ occurs along different directions. The first one is a lateral translation along $x$ direction with maximum displacement $U_{x}=\frac{3}{8}\left(R_{\mathrm{e}}-R_{\mathrm{i}}\right)$. The second one is composed by an axial translation $\left(U_{y}=R_{\mathrm{i}}\right)$ and rotation $\left(\theta_{y}=45^{\circ}\right)$. The 


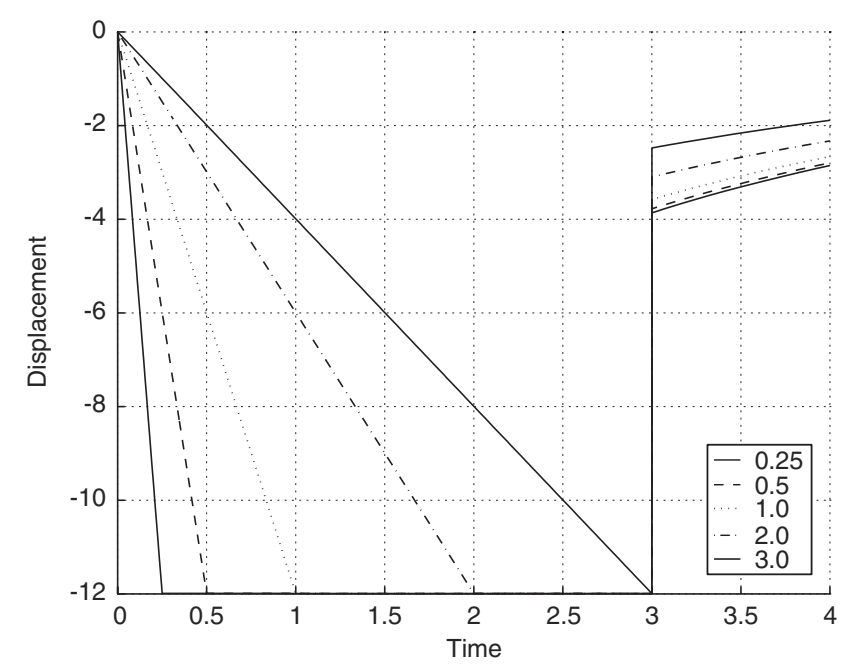

Figure 17. Displacement $u_{z}$ of interior point below punch tip.

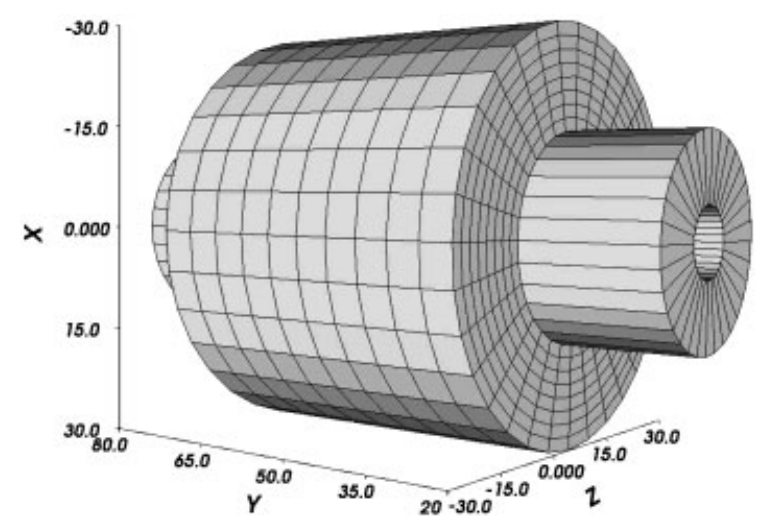

Figure 18. Support. Undeformed configuration.

dimensions of the viscoelastic support are $R_{\mathrm{i}}=15 \mathrm{~mm}, R_{\mathrm{e}}=30 \mathrm{~mm}$ and $L=50 \mathrm{~mm}$. Figure 19 shows the maximum amplitude of deformations for a lateral motion while Figure 20 illustrates the case of axial (rotation plus translation) motion. The isochoric nature of the material shows up in Figure 19 where the compressed and tractioned sides of the cylinder deform accordingly to preserve this constraint. Two material models were run, both sharing the same constitutive characteristics in the small strain regime.

Case 1: Ogden model for both spring potentials $\varphi$ and $\varphi^{\mathrm{e}}\left(\mu_{i}^{\mathrm{e}}=4 \mu_{i}\right): \mu_{1}=3.5 ; \mu_{2}=0.011$; $\mu_{3}=0.0015$ and $\alpha_{1}=0.1 ; \alpha_{2}=2.0 ; \alpha_{3}=9\left(\mu_{\mathrm{eq}}=\sum_{i} \frac{1}{2} \mu_{i} \alpha_{i}=0.19275 \mathrm{MPa}\right)$. The viscous potential $\psi$ is of the Hencky type with $\eta^{\mathrm{v}}=7.69(\tau=0.4 \mathrm{~s})$. Potential $\phi$ is null. 

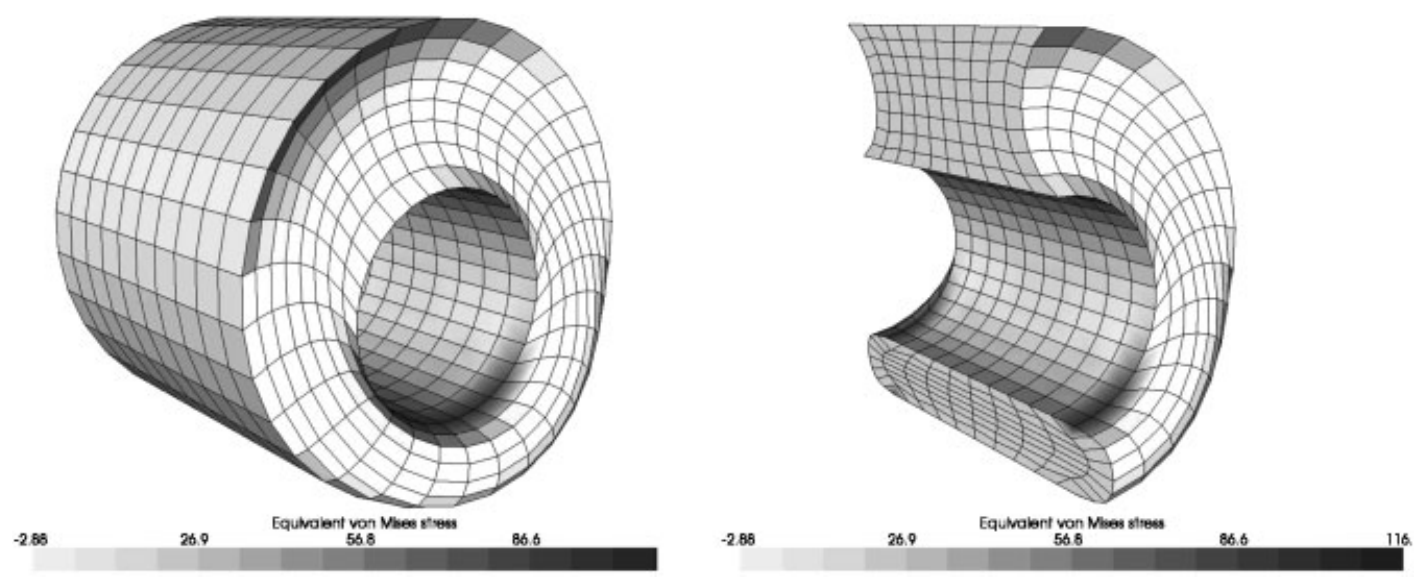

Figure 19. Support. Case 1: deformed configuration for lateral edge motion.
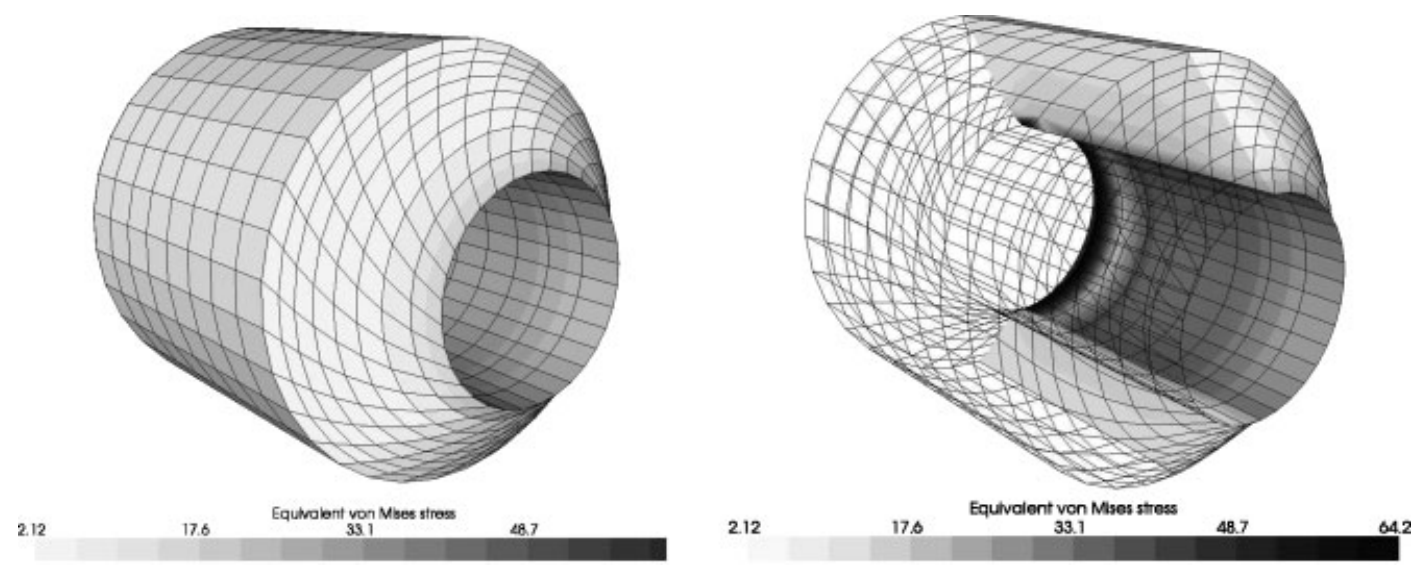

Figure 20. Support. Case 2: deformed configuration for axial edge motion.

Case 2: All potentials of the Hencky type: $\mu=0.19275 \mathrm{MPa}, \mu^{\mathrm{e}}=4 \mu, \eta^{\mathrm{v}}=\tau \mu^{\mathrm{e}}, \tau=0.4 \mathrm{~s}$. Potential $\phi$ is null.

Figures 21-22 show the hysteretical loops in force/displacement for the lateral and axial motions, respectively. These graphics clearly show the differences induced by the model, which are accentuated in large strain regimes.

\section{CONCLUDING REMARKS}

This paper presents a general set of viscoelastic constitutive models based on a variational framework that provides an appropriate mathematical structure for further applications like, for example, error estimation (see a detailed study on this issue in Reference [12]). Among others, 


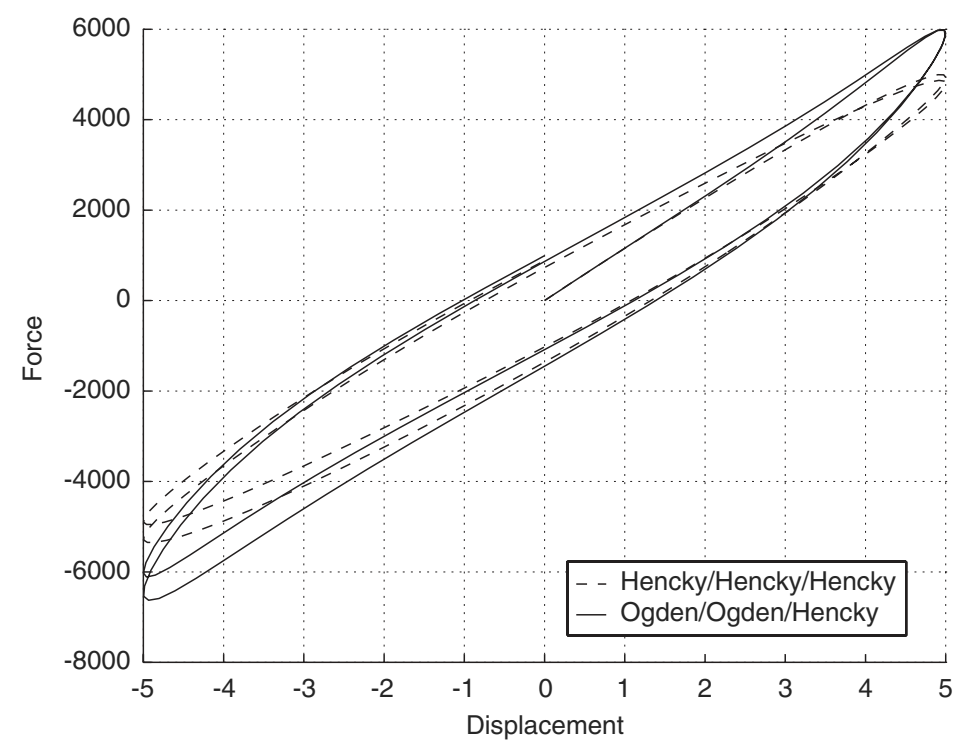

Figure 21. Support. Case 1: lateral force $F_{x}$ versus displacement $u_{x}$.

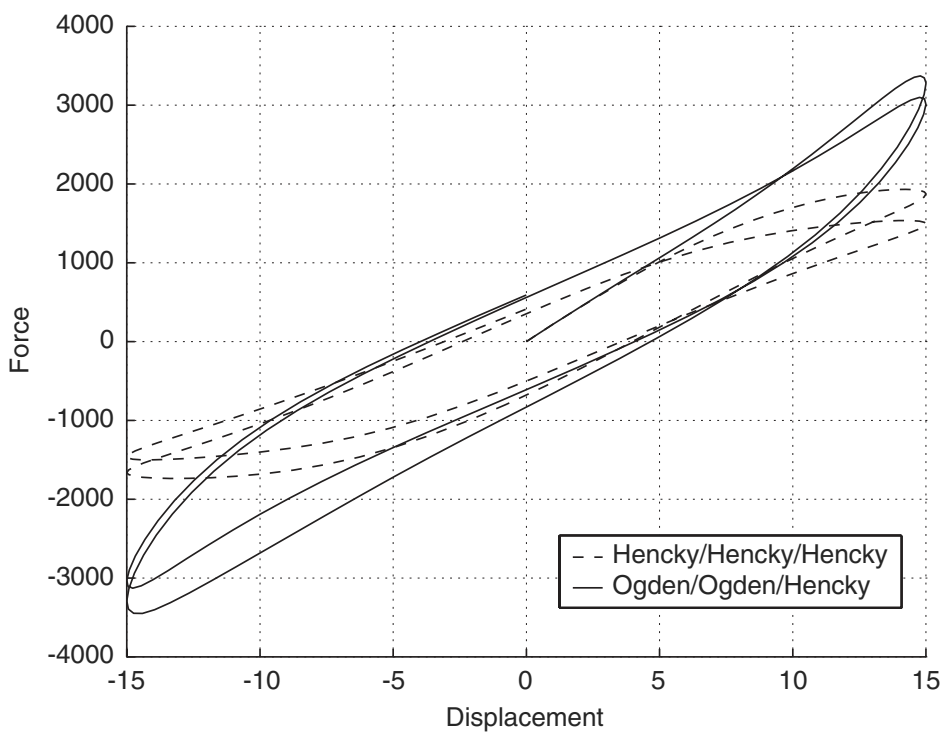

Figure 22. Support. Case 2: axial force $F_{y}$ versus displacement $u_{y}$. 
the following characteristics of this approach should be noted:

- It is capable of reproducing other models in literature according to the specific viscoelastic law chosen;

- No theoretical restrictions are put on the size of perturbations away from the equilibrium positions and they can be properly treated with appropriate choices of dissipative functionals;

- The theoretical and operational (implementation) background is stated for general isotropic constitutive functions depending on eigenvalues of strains and strain rates. As a consequence, most of the implementation effort, including stress updates and tangent matrix is carried out at a generic level with no relation to a specific isotropic law (potential). This last definition can be performed practically at user level by the choice of the corresponding potential and derivatives expressions (see, for example, (74), (75) or (79) $-(82))$.

- Stress and strain updates require (in the general case) the solution of a three-equation non-linear system to determine the eigenvalues of viscoelastic increments (eigenprojections are defined by the predictor state). This non-linear system is analytically invertible and, thus, computationally inexpensive. Moreover, the material tensor is calculated by means of a simple closed form expression. From this remark and from the behaviour shown by the numerical experiments, we observed that the performance of the constitutive update based on the present expressions is, in general, comparable to that of a classic radial-return plastic von Mises-based problem (with non-linear hardening).

- More than a single Maxwell branch are frequently employed to describe real experimental viscoelastic curves. The proposed model is, as already shown, able to include those branches with the corresponding cost of new parameters to be identified. We also note that the present formulation allows the use of different non-linear models for each component of the Maxwell branch, starting from classical two-parameter models to more sophisticated ones. A question that deserves further studies is related to the number of Maxwell branches needed to describe real viscoelastic curves depending on the features of the models defined in each of them.

- Extension to non-isotropic materials presents, a priori, no mathematical difficulties and is the subject of future developments.

\section{APPENDIX A}

Minimization of $\Psi$ with respect to $\mathbf{M}_{a}^{\mathrm{v}}$

The argument of the minimum of potential $\Psi\left(\Delta q_{j}^{\mathrm{v}}, \mathbf{M}_{j}^{\mathrm{v}}\right)$ must satisfy the usual constraints for eigenprojections and the traceless property provided by the eigenvalues:

$$
\begin{aligned}
\Delta q_{j}^{\mathrm{v}} \in K_{Q} & =\left\{p_{j} \in \mathbb{R}^{1}: p_{1}+p_{2}+p_{3}=0\right\} \\
\mathbf{M}_{j}^{\mathrm{v}} \in K_{M} & =\left\{\mathbf{N}_{j} \in \operatorname{Sym}: \mathbf{N}_{j} \cdot \mathbf{N}_{j}=1, \quad \mathbf{N}_{i} \cdot \mathbf{N}_{j}=0, \quad i \neq j\right\}
\end{aligned}
$$

In order to enforce them, the optimality condition (51) is computed with the aid of a Lagrangian function that adds to the original potential all the considered equality constraints multiplied by 
corresponding Lagrangian multipliers:

$$
\begin{aligned}
\mathscr{L}\left(\varepsilon_{j}^{\mathrm{e}}, d_{j}^{\mathrm{v}}, \lambda\right)= & \Delta \varphi^{\mathrm{e}}\left(\varepsilon_{j}^{\mathrm{e}}\right)+\Delta t \psi\left(d_{j}^{\mathrm{e}}\right) \\
& +\lambda_{1}\left(\mathbf{M}_{a}^{\mathrm{v}} \cdot \mathbf{M}_{a}^{\mathrm{v}}-1\right)+\lambda_{2}\left(\mathbf{M}_{a}^{\mathrm{v}} \cdot \mathbf{M}_{b}^{\mathrm{v}}\right)+\lambda_{3}\left(\mathbf{M}_{a}^{\mathrm{v}} \cdot \mathbf{M}_{c}^{\mathrm{v}}\right) \\
& +\lambda_{4}\left(\mathbf{M}_{b}^{\mathrm{v}} \cdot \mathbf{M}_{b}^{\mathrm{v}}-1\right)+\lambda_{5}\left(\mathbf{M}_{b}^{\mathrm{v}} \cdot \mathbf{M}_{c}^{\mathrm{v}}\right)+\lambda_{6}\left(\mathbf{M}_{c}^{\mathrm{v}} \cdot \mathbf{M}_{c}^{\mathrm{v}}-1\right) \\
& +\lambda\left(d_{a}^{\mathrm{v}}+d_{b}^{\mathrm{v}}+d_{c}^{\mathrm{v}}\right)
\end{aligned}
$$

We also recall the relations

$$
\begin{gathered}
\mathbf{D}^{\mathrm{v}}=\sum_{j=1}^{3} d_{j}^{\mathrm{v}} \mathbf{M}_{j}^{\mathrm{v}}, \quad d_{j}=\frac{\Delta q_{j}^{\mathrm{v}}}{\Delta t}, \quad \frac{\partial d_{a}^{\mathrm{v}}}{\partial \mathbf{D}^{\mathrm{v}}}=\mathbf{M}_{a}^{\mathrm{v}} \\
\varepsilon^{\mathrm{e}}=\sum_{j=1}^{3} \varepsilon_{j}^{\mathrm{e}} \mathbf{E}_{j}^{\mathrm{e}}=\varepsilon^{\mathrm{pr}}-\Delta t \mathbf{D}^{\mathrm{v}}, \quad \frac{\partial \varepsilon_{a}^{\mathrm{e}}}{\partial \varepsilon^{\mathrm{e}}}=\mathbf{E}_{a}^{\mathrm{e}}
\end{gathered}
$$

The optimality condition with respect to direction $\mathbf{M}_{a}$ is

$$
\begin{aligned}
\frac{\partial \mathscr{L}}{\partial \mathbf{M}_{a}^{\mathrm{v}}}[\delta \mathbf{M}]= & \left(\frac{\partial \Delta \varphi^{\mathrm{e}}}{\partial \mathbf{M}_{a}^{\mathrm{v}}}+\Delta t \frac{\partial \Delta \psi}{\partial \mathbf{M}_{a}^{\mathrm{v}}}\right)[\delta \mathbf{M}]+2 \lambda_{1}\left(\mathbf{M}_{a}^{\mathrm{v}} \cdot \delta \mathbf{M}\right)+\lambda_{2}\left(\mathbf{M}_{b}^{\mathrm{v}} \cdot \delta \mathbf{M}\right)+\lambda_{3}\left(\mathbf{M}_{c}^{\mathrm{v}} \cdot \delta \mathbf{M}\right) \\
& +\lambda\left(\frac{\partial d_{a}^{\mathrm{v}}}{\partial \mathbf{D}^{\mathrm{v}}} \frac{\partial \mathbf{D}^{\mathrm{v}}}{\partial \mathbf{M}_{a}^{\mathrm{v}}}\right)[\delta \mathbf{M}]=0 \quad \forall \delta \mathbf{M} \in \operatorname{Sym}
\end{aligned}
$$

where

$$
\begin{aligned}
\frac{\partial \Delta \varphi^{\mathrm{e}}}{\partial \mathbf{M}_{a}^{\mathrm{v}}} & =\sum_{j=1}^{3} \frac{\partial \Delta \varphi^{\mathrm{e}}}{\partial \varepsilon_{j}^{\mathrm{e}}} \frac{\partial \varepsilon_{j}^{\mathrm{e}}}{\partial \varepsilon^{\mathrm{e}}} \frac{\partial \varepsilon^{\mathrm{e}}}{\partial \mathbf{M}_{a}^{\mathrm{v}}}=-\left(\sum_{j=1}^{3} \frac{\partial \Delta \varphi^{\mathrm{e}}}{\partial \varepsilon_{j}^{\mathrm{e}}} \mathbf{E}_{j}^{\mathrm{e}}\right) \Delta q_{a}^{\mathrm{v}} \\
\frac{\partial \Delta \psi}{\partial \mathbf{M}_{a}^{\mathrm{v}}} & =\sum_{j=1}^{3} \frac{\partial \Delta \psi}{\partial d_{j}^{\mathrm{v}}} \frac{\partial d_{j}^{\mathrm{v}}}{\partial \mathbf{D}^{\mathrm{v}}} \frac{\partial \mathbf{D}^{\mathrm{v}}}{\partial \mathbf{M}_{a}^{\mathrm{v}}}=\left(\sum_{j=1}^{3} \frac{\partial \Delta \psi}{\partial d_{j}^{\mathrm{v}}} \mathbf{M}_{j}^{\mathrm{v}}\right) \frac{\Delta q_{a}^{\mathrm{v}}}{\Delta t} \\
\frac{\partial d_{a}^{\mathrm{v}}}{\partial \mathbf{D}^{\mathrm{v}}} \frac{\partial \mathbf{D}^{\mathrm{v}}}{\partial \mathbf{M}_{a}^{\mathrm{v}}} & =d_{a}^{\mathrm{v}} \mathbf{M}_{a}^{\mathrm{v}}(\text { no sum on index a) } \\
\left(\frac{\partial \Delta \varphi^{\mathrm{e}}}{\partial \mathbf{M}_{a}^{\mathrm{v}}}+\Delta t \frac{\partial \Delta \psi}{\partial \mathbf{M}_{a}^{\mathrm{v}}}\right) & =\Delta q_{a}^{\mathrm{v}} \underbrace{\left[\left(\sum_{j=1}^{3} \frac{\partial \Delta \psi}{\partial d_{j}^{\mathrm{v}}} \mathbf{M}_{j}^{\mathrm{v}}\right)-\left(\sum_{j=1}^{3} \frac{\partial \Delta \varphi^{\mathrm{e}}}{\partial \varepsilon_{j}^{\mathrm{e}}} \mathbf{E}_{j}^{\mathrm{e}}\right)\right]}_{\mathbf{A}}=\Delta q_{a}^{\mathrm{v}} \mathbf{A}
\end{aligned}
$$


Substituting these results in (A2), and taking $\delta \mathbf{M}$ equal to $\mathbf{M}_{a}^{\mathrm{v}}, \mathbf{M}_{b}^{\mathrm{v}}, \mathbf{M}_{c}^{\mathrm{v}}$ respectively, we obtain the conditions

$$
\begin{aligned}
2 \lambda_{1}+\lambda d_{a}^{\mathrm{v}} & =-\Delta q_{a}^{\mathrm{v}} \mathbf{A} \cdot \mathbf{M}_{a}^{\mathrm{v}} \\
\lambda_{2} & =-\Delta q_{a}^{\mathrm{v}} \mathbf{A} \cdot \mathbf{M}_{b}^{\mathrm{v}} \\
\lambda_{3} & =-\Delta q_{a}^{\mathrm{v}} \mathbf{A} \cdot \mathbf{M}_{c}^{\mathrm{v}}
\end{aligned}
$$

Substituting them in (A2),

$\Delta q_{a}^{\mathrm{v}}\left[\mathbf{A} \cdot \delta \mathbf{M}-\left(\mathbf{A} \cdot \mathbf{M}_{a}^{\mathrm{v}}\right)\left(\mathbf{M}_{a}^{\mathrm{v}} \cdot \delta \mathbf{M}\right)-\left(\mathbf{A} \cdot \mathbf{M}_{b}^{\mathrm{v}}\right)\left(\mathbf{M}_{b}^{\mathrm{v}} \cdot \delta \mathbf{M}\right)-\left(\mathbf{A} \cdot \mathbf{M}_{c}^{\mathrm{v}}\right)\left(\mathbf{M}_{c}^{\mathrm{v}} \cdot \delta \mathbf{M}\right)\right]=0 \quad \forall \delta \mathbf{M} \in \operatorname{Sym}$ or, equivalently,

$$
\overbrace{\left[\square-\left(\mathbf{M}_{a}^{\mathrm{v}} \otimes \mathbf{M}_{a}^{\mathrm{v}}\right)-\left(\mathbf{M}_{b}^{\mathrm{v}} \otimes \mathbf{M}_{b}^{\mathrm{v}}\right)-\left(\mathbf{M}_{c}^{\mathrm{v}} \otimes \mathbf{M}_{c}^{\mathrm{v}}\right)\right]}^{\mathbb{B}} \mathbf{A}=\mathbf{0}
$$

Substituting the tensor $\mathbf{A}$ in this last equation it is simple to verify that

$$
\begin{aligned}
\mathbb{B}\left(\sum_{j=1}^{3} \frac{\partial \Delta \psi}{\partial d_{j}^{\mathrm{v}}} \mathbf{M}_{j}^{\mathrm{v}}\right)= & \mathbf{0} \\
\mathbb{B}\left(\sum_{j=1}^{3} \frac{\partial \Delta \varphi^{\mathrm{e}}}{\partial \varepsilon_{j}^{\mathrm{e}}} \mathbf{E}_{j}^{\mathrm{e}}\right)= & \sum_{j=1}^{3} \frac{\partial \Delta \varphi^{\mathrm{e}}}{\partial \varepsilon_{j}^{\mathrm{e}}} \mathbf{E}_{j}^{\mathrm{e}}-\sum_{j=1}^{3} \frac{\partial \Delta \varphi^{\mathrm{e}}}{\partial \varepsilon_{j}^{\mathrm{e}}}\left(\mathbf{E}_{j}^{\mathrm{e}} \cdot \mathbf{M}_{a}^{\mathrm{v}}\right) \mathbf{M}_{a}^{\mathrm{v}} \\
& -\sum_{j=1}^{3} \frac{\partial \Delta \varphi^{\mathrm{e}}}{\partial \varepsilon_{j}^{\mathrm{e}}}\left(\mathbf{E}_{j}^{\mathrm{e}} \cdot \mathbf{M}_{b}^{\mathrm{v}}\right) \mathbf{M}_{b}^{\mathrm{v}}-\sum_{j=1}^{3} \frac{\partial \Delta \varphi^{\mathrm{e}}}{\partial \varepsilon_{j}^{\mathrm{e}}}\left(\mathbf{E}_{j}^{\mathrm{e}} \cdot \mathbf{M}_{c}^{\mathrm{v}}\right) \mathbf{M}_{c}^{\mathrm{v}}
\end{aligned}
$$

The last equation is zero if we take $\mathbf{E}_{j}^{\mathrm{e}}=\mathbf{M}_{j}^{\mathrm{v}}$.

Minimization of $\Psi$ with respect to $\Delta q_{a}^{\mathrm{v}}$

Once the directions $\mathbf{M}_{i}^{\mathrm{v}}$ are computed, the minimization with respect to $\Delta q_{i}^{\mathrm{v}}$ or equivalently to $d_{i}^{\mathrm{v}}=\Delta q_{i}^{\mathrm{v}} / \Delta t$ should be performed. Considering that $\varepsilon_{i}^{\mathrm{e}}=\varepsilon_{i}^{\mathrm{pr}}-\Delta q_{i}^{\mathrm{v}}$, and the fact that constraints $\varepsilon_{1}^{\mathrm{e}}+\varepsilon_{2}^{\mathrm{e}}+\varepsilon_{3}^{\mathrm{e}}=d_{1}^{\mathrm{v}}+d_{2}^{\mathrm{v}}+d_{3}^{\mathrm{v}}=0$, we have the following optimality conditions:

$$
\begin{aligned}
r_{i} & =-\frac{\partial \mathscr{L}}{\partial \Delta q_{i}^{\mathrm{v}}}=\frac{\partial \mathscr{L}}{\partial \varepsilon_{i}^{\mathrm{e}}}=\frac{\partial \varphi^{\mathrm{e}}}{\partial \varepsilon_{i}^{\mathrm{e}}}+\Delta t \frac{\partial \psi}{\partial d_{i}^{\mathrm{v}}} \frac{\partial d_{i}^{\mathrm{v}}}{\partial \varepsilon_{i}^{\mathrm{e}}}+\lambda \\
& =\frac{\partial \varphi^{\mathrm{e}}}{\partial \varepsilon_{i}^{\mathrm{e}}}-\frac{\partial \psi}{\partial d_{i}^{\mathrm{v}}}+\lambda=0, \quad i=1,2,3 \\
r_{4} & =\varepsilon_{1}^{\mathrm{e}}+\varepsilon_{2}^{\mathrm{e}}+\varepsilon_{3}^{\mathrm{e}}=0
\end{aligned}
$$

Using the Newton method to find the roots $\mathbf{x}=\left\{\varepsilon_{1}^{\mathrm{e}}, \varepsilon_{2}^{\mathrm{e}}, \varepsilon_{3}^{\mathrm{e}}, \lambda\right\}$, we have the recursive formula

$$
\mathbf{x}^{k+1}=\mathbf{x}^{k}-\mathbf{K}^{k-1} \mathbf{r}^{k}
$$


or

$$
\mathbf{K}^{k} \Delta \mathbf{x}=-\mathbf{r}^{k}, \quad \mathbf{x}^{k+1}=\mathbf{x}^{k}+\Delta \mathbf{x}
$$

Further simplifications can be made if we assume that

$$
\varphi^{\mathrm{e}}\left(\varepsilon_{i}^{\mathrm{e}}\right)=\sum_{i=1}^{3} w^{\mathrm{e}}\left(\varepsilon_{i}^{\mathrm{e}}\right), \quad \psi\left(d_{i}^{\mathrm{v}}\right)=\sum_{i=1}^{3} w^{\mathrm{v}}\left(d_{i}^{\mathrm{v}}\right)
$$

as is the case of the Hencky or Ogden model proposed. Thus, we have

$$
\begin{aligned}
\mathbf{K} & =\left[\begin{array}{cccc}
K_{11} & 0 & 0 & 1 \\
0 & K_{22} & 0 & 1 \\
0 & 0 & K_{33} & 1 \\
1 & 1 & 1 & 0
\end{array}\right] \\
K_{i i} & =\frac{\partial^{2} \varphi^{\mathrm{e}}}{\partial \varepsilon_{i}^{\mathrm{e}} \partial \varepsilon_{i}^{\mathrm{e}}}+\frac{1}{\Delta t} \frac{\partial \psi}{\partial d_{i}^{\mathrm{v}} \partial d_{i}^{\mathrm{v}}}, \quad i=1,2,3 . \text { (no index sum) }
\end{aligned}
$$

In this case, after some algebra, we have the following explicit expression for the Newton update:

$$
\begin{aligned}
\Delta \varepsilon_{i}^{\mathrm{e}} & =-\left(r_{i}+\Delta \lambda\right) / K_{i i} \\
\Delta \lambda & =\left(r_{4}-\sum_{j=1}^{3} \frac{r_{j}}{K_{j j}}\right)\left(\sum_{s=1}^{3} \frac{1}{K_{s s}}\right)^{-1}
\end{aligned}
$$

Derivatives of $\varphi, \phi$ and $\varphi^{\mathrm{e}}$ with respect to $\hat{\mathbf{C}}_{n+1}$

The derivative of $\phi$ is straightforward:

$$
\begin{aligned}
\hat{\mathbf{C}}_{n+1} & =\sum_{j=1}^{3} c_{j} \mathbf{E}_{j} \\
\frac{\partial \varphi}{\partial \hat{\mathbf{C}}_{n+1}} & =\sum_{j=1}^{3} \frac{\partial \varphi}{\partial c_{j}} \frac{\partial c_{j}}{\partial \hat{\mathbf{C}}_{n+1}}=\sum_{j=1}^{3} \frac{\partial \varphi}{\partial c_{j}} \mathbf{E}_{j}
\end{aligned}
$$

Considering that

$$
\begin{aligned}
\Delta \hat{\mathbf{C}} & =(\Delta \hat{\mathbf{F}})^{T} \Delta \hat{\mathbf{F}}=\hat{\mathbf{F}}_{n}^{-\mathrm{T}} \hat{\mathbf{C}}_{n+1} \hat{\mathbf{F}}_{n}^{-1} \\
\mathbf{D} & =\sum_{j=1}^{3} d_{j} \mathbf{M}_{j}=\frac{1}{2 \Delta t} \ln (\Delta \hat{\mathbf{C}})=\frac{1}{2 \Delta t} \sum_{j=1}^{3} \ln \left(\Delta c_{j}\right) \mathbf{M}_{j} \\
d_{j} & =\frac{1}{2 \Delta t} \ln \left(\Delta c_{j}\right), \quad \frac{\partial d_{j}}{\Delta c_{j}}=\frac{1}{2 \Delta t \Delta c_{j}}
\end{aligned}
$$


the derivative of $\phi$ is

$$
\begin{aligned}
\frac{\partial \phi}{\partial \hat{\mathbf{C}}_{n+1}}[\delta \mathbf{C}] & =\frac{\partial \phi}{\partial \Delta \hat{\mathbf{C}}} \cdot \frac{\partial \Delta \hat{\mathbf{C}}}{\partial \hat{\mathbf{C}}_{n+1}}[\delta \mathbf{C}] \\
& =\frac{\partial \phi}{\partial \Delta \hat{\mathbf{C}}} \cdot \hat{\mathbf{F}}_{n}^{-\mathrm{T}}\left(\frac{\partial \hat{\mathbf{C}}_{n+1}}{\partial \hat{\mathbf{C}}_{n+1}}[\delta \mathbf{C}]\right) \hat{\mathbf{F}}_{n}^{-1}=\frac{\partial \phi}{\partial \Delta \hat{\mathbf{C}}} \cdot \hat{\mathbf{F}}_{n}^{-\mathrm{T}} \frac{1}{2}\left(\delta \mathbf{C}+\delta \mathbf{C}^{T}\right) \hat{\mathbf{F}}_{n}^{-1} \\
& =\hat{\mathbf{F}}_{n}^{-1} \frac{\partial \phi}{\partial \Delta \hat{\mathbf{C}}} \hat{\mathbf{F}}_{n}^{-\mathrm{T}} \cdot \delta \mathbf{C} \\
\frac{\partial \phi}{\partial \Delta \hat{\mathbf{C}}} & =\sum_{j=1}^{3} \frac{\partial \phi}{\partial d_{j}} \frac{\partial d_{j}}{\partial \Delta c_{j}} \frac{\partial \Delta c_{j}}{\partial \Delta \hat{\mathbf{C}}}=\sum_{j=1}^{3} \frac{\partial \phi}{\partial d_{j}} \frac{1}{2 \Delta t \Delta c_{j}} \mathbf{M}_{j}
\end{aligned}
$$

In order to compute the derivative $\varphi^{\mathrm{e}}$ we use the relations

$$
\begin{aligned}
\hat{\mathbf{C}}_{n+1}^{\mathrm{e}} & =\hat{\mathbf{F}}_{n+1}^{\mathrm{eT}} \hat{\mathbf{F}}_{n+1}^{\mathrm{e}}=\mathbf{F}_{n+1}^{\mathrm{v}-\mathrm{T}} \hat{\mathbf{C}}_{n+1} \mathbf{F}_{n+1}^{\mathrm{v}-1} \\
\hat{\mathbf{C}}^{\mathrm{pr}} & =\hat{\mathbf{F}}^{\mathrm{prT}} \hat{\mathbf{F}}^{\mathrm{pr}}=\mathbf{F}_{n}^{\mathrm{v}-\mathrm{T}} \hat{\mathbf{C}}_{n+1} \mathbf{F}_{n}^{\mathrm{v}-1}
\end{aligned}
$$

and by operating in an analogous form as in (A4) we obtain

$$
\begin{aligned}
\frac{\partial \varphi^{\mathrm{e}}}{\partial \hat{\mathbf{C}}_{n+1}} & =\mathbf{F}_{n+1}^{\mathrm{v}-1} \frac{\partial \varphi^{\mathrm{e}}}{\partial \hat{\mathbf{C}}_{n+1}^{\mathrm{e}}} \mathbf{F}_{n+1}^{\mathrm{v}-\mathrm{T}} \\
& =\mathbf{F}_{n}^{\mathrm{v}-1} \frac{\partial \varphi^{\mathrm{e}}}{\partial \hat{\mathbf{C}}^{\mathrm{pr}}} \mathbf{F}_{n}^{\mathrm{v}-\mathrm{T}}
\end{aligned}
$$

where,

$$
\begin{aligned}
\frac{\partial \varphi^{\mathrm{e}}}{\partial \hat{\mathbf{C}}_{n+1}^{\mathrm{e}}} & =\sum_{j=1}^{3} \frac{\partial \varphi^{\mathrm{e}}}{\partial c_{j}^{\mathrm{e}}} \frac{\partial c_{j}^{\mathrm{e}}}{\partial \hat{\mathbf{C}}_{n+1}^{\mathrm{e}}}=\sum_{j=1}^{3} \frac{\partial \varphi^{\mathrm{e}}}{\partial c_{j}^{\mathrm{e}}} \mathbf{E}_{j}^{\mathrm{e}} \\
\frac{\partial \varphi^{\mathrm{e}}}{\partial \hat{\mathbf{C}}^{\mathrm{pr}}} & =\sum_{j=1}^{3} \frac{\partial \varphi^{\mathrm{e}}}{\partial c_{j}^{\mathrm{pr}}} \frac{\partial c_{j}^{\mathrm{pr}}}{\partial \hat{\mathbf{C}}_{n+1}^{\mathrm{e}}}=\sum_{j=1}^{3} \frac{\partial \varphi^{\mathrm{e}}}{\partial c_{j}^{\mathrm{pr}}} \mathbf{E}_{j}^{\mathrm{pr}}
\end{aligned}
$$

Hencky model: derivatives of $\varphi, \phi$ and $\varphi^{\mathrm{e}}$

From the definition of the potentials,

$$
\begin{aligned}
& \frac{\partial \varphi}{\partial \varepsilon_{j}}=2 \mu \varepsilon_{j}, \quad \frac{\partial \phi}{\partial d_{j}}=2 \eta d_{j}, \quad \frac{\partial \varphi^{\mathrm{e}}}{\partial \varepsilon_{j}^{\mathrm{e}}}=2 \mu^{\mathrm{e}} \varepsilon_{j}^{\mathrm{e}}, \quad \frac{\partial \psi}{\partial d_{j}^{\mathrm{v}}}=2 \mu^{\mathrm{v}} d_{j}^{\mathrm{v}} \\
& \frac{\partial^{2} \varphi}{\left(\partial \varepsilon_{j}\right)^{2}}=2 \mu, \quad \frac{\partial^{2} \phi}{\left(\partial d_{j}\right)^{2}}=2 \eta, \quad \frac{\partial^{2} \varphi^{\mathrm{e}}}{\left(\partial \varepsilon_{j}^{\mathrm{e}}\right)^{2}}=2 \mu^{\mathrm{e}}, \quad \frac{\partial^{2} \psi}{\left(\partial d_{j}^{\mathrm{v}}\right)^{2}}=2 \mu^{\mathrm{v}}
\end{aligned}
$$


Ogden model: derivatives of $\varphi, \phi$ and $\varphi^{\mathrm{e}}$

Recalling that $c_{j}=\exp \left(2 \varepsilon_{j}\right), c_{j}^{\mathrm{e}}=\exp \left(2 \varepsilon_{j}^{\mathrm{e}}\right), \exp \left(d_{j}\right)=\left(\Delta c_{j}\right)^{1 / 2 \Delta t}$, we have for the Ogden model

$$
\begin{aligned}
\varphi & =\sum_{j=1}^{3} \sum_{p=1}^{N} \frac{\mu_{p}}{\alpha_{p}}\left(\left[\exp \left(\varepsilon_{j}\right)\right]^{\alpha_{p}}-1\right)=\sum_{j=1}^{3} \sum_{p=1}^{N} \frac{\mu_{p}}{\alpha_{p}}\left(\left(c_{j}\right)^{\alpha_{p} / 2}-1\right) \\
\varphi^{\mathrm{e}} & =\sum_{j=1}^{3} \sum_{p=1}^{N} \frac{\mu_{p}^{\mathrm{e}}}{\alpha_{p}}\left(\left[\exp \left(\varepsilon_{j}^{\mathrm{e}}\right)\right]^{\alpha_{p}}-1\right)=\sum_{j=1}^{3} \sum_{p=1}^{N} \frac{\mu_{p}^{\mathrm{e}}}{\alpha_{p}}\left(\left(c_{j}^{\mathrm{e}}\right)^{\alpha_{p} / 2}-1\right) \\
\phi & =\sum_{j=1}^{3} \sum_{p=1}^{N} \frac{\eta_{p}}{\alpha_{p}}\left(\left[\exp \left(d_{j}\right)\right]^{\alpha_{p}}-1\right)=\sum_{j=1}^{3} \sum_{p=1}^{N} \frac{\eta_{p}}{\alpha_{p}}\left(\left(\Delta c_{j}\right)^{\alpha_{p} / 2 \Delta t}-1\right)
\end{aligned}
$$

Deriving, we have

$$
\begin{aligned}
& \frac{\partial \varphi}{\partial \varepsilon_{j}}=\sum_{p=1}^{N} \mu_{p}\left[\exp \left(\varepsilon_{j}\right)\right]^{\alpha_{p}}, \quad \frac{\partial \phi}{\partial d_{j}}=\sum_{p=1}^{N} \eta_{p}\left[\exp \left(d_{j}\right)\right]^{\alpha_{p}} \\
& \frac{\partial \varphi^{\mathrm{e}}}{\partial \varepsilon_{j}^{\mathrm{e}}}=\sum_{p=1}^{N} \mu_{p}^{\mathrm{e}}\left[\exp \left(\varepsilon_{j}^{\mathrm{e}}\right)\right]^{\alpha_{p}}, \quad \frac{\partial \psi}{\partial d_{j}^{\mathrm{v}}}=\sum_{p=1}^{N} \eta_{p}^{\mathrm{v}}\left[\exp \left(d_{j}^{\mathrm{v}}\right)\right]^{\alpha_{p}} \\
& \frac{\partial^{2} \varphi}{\left(\partial \varepsilon_{j}\right)^{2}}=\sum_{p=1}^{N} \mu_{p} \alpha_{p}\left[\exp \left(\varepsilon_{j}\right)\right]^{\alpha_{p}}, \quad \frac{\partial^{2} \phi}{\left(\partial d_{j}\right)^{2}}=\sum_{p=1}^{N} \eta_{p} \alpha_{p}\left[\exp \left(d_{j}\right)\right]^{\alpha_{p}} \\
& \frac{\partial^{2} \varphi^{\mathrm{e}}}{\left(\partial \varepsilon_{j}^{\mathrm{e}}\right)^{2}}=\sum_{p=1}^{N} \mu_{p}^{\mathrm{e}} \alpha_{p}\left[\exp \left(\varepsilon_{j}^{\mathrm{e}}\right)\right]^{\alpha_{p}}, \quad \frac{\partial^{2} \psi}{\left(\partial d_{j}^{\mathrm{v}}\right)^{2}}=\sum_{p=1}^{N} \eta_{p}^{\mathrm{v}} \alpha_{p}\left[\exp \left(d_{j}^{\mathrm{v}}\right)\right]^{\alpha_{p}}
\end{aligned}
$$

\section{Material tensors}

Using the relation $\Delta \hat{\mathbf{C}}=\hat{\mathbf{F}}_{n}^{-\mathrm{T}} \hat{\mathbf{C}}_{n+1} \hat{\mathbf{F}}_{n}^{-1}=\mathbf{f}^{n \mathrm{~T}} \hat{\mathbf{C}} \mathbf{f}^{n}$, we have that (in this particular expression we use Einstein's notation for summation on indices $p, q, m, t, u, v, r, s)$ :

$$
\begin{aligned}
& \frac{\partial}{\partial \hat{\mathbf{C}}_{k l}}\left(\frac{\partial \phi}{\partial \hat{\mathbf{C}}_{i j}}\right)=\frac{\partial}{\partial \Delta \hat{\mathbf{C}}_{p q}} \frac{\partial \phi}{\partial \Delta \hat{\mathbf{C}}_{m t}} \frac{\partial \Delta \hat{\mathbf{C}}_{m t}}{\partial \hat{\mathbf{C}}_{i j}} \frac{\partial \Delta \hat{\mathbf{C}}_{p q}}{\partial \hat{\mathbf{C}}_{k l}}
\end{aligned}
$$

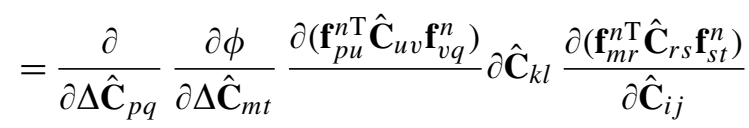

$$
\begin{aligned}
& =\frac{\partial}{\partial \Delta \hat{\mathbf{C}}_{p q}} \frac{\partial \phi}{\partial \Delta \hat{\mathbf{C}}_{m t}} \mathbf{f}_{p u}^{n \mathrm{~T}} \Pi_{u v k l} \mathbf{f}_{v q}^{n} \mathbf{f}_{m r}^{n \mathrm{~T}} \Pi_{r s i j} \mathbf{f}_{s t}^{n} \\
& =\mathbf{f}_{r m}^{n} \mathbf{f}_{s t}^{n} \frac{\partial \phi}{\partial \Delta \hat{\mathbf{C}}_{m t} \partial \Delta \hat{\mathbf{C}}_{p q}} \mathbf{f}_{u p}^{n} \mathbf{f}_{v q}^{n} \mathbb{I}_{u v k l} \mathbb{I}_{r i j}
\end{aligned}
$$




$$
\begin{aligned}
& =\mathbf{f}_{i m}^{n} \mathbf{f}_{j t}^{n} \frac{\partial \phi}{\partial \Delta \hat{\mathbf{C}}_{m t} \partial \Delta \hat{\mathbf{C}}_{p q}} \mathbf{f}_{k p}^{n} \mathbf{f}_{l q}^{n}=\mathscr{C}_{i j k l}^{\phi} \\
\mathscr{C}_{i j k l}^{\phi} & =\mathscr{C}_{k l i j}^{\phi}, \quad \mathscr{C}_{i j k l}^{\phi}=\mathscr{C}_{j i k l}^{\phi}, \mathbb{I}_{i j k l}=\frac{1}{2}\left(\delta_{i k} \delta_{j l}+\delta_{i l} \delta_{j k}\right)
\end{aligned}
$$

The total derivative of $\varphi^{\mathrm{e}}$ with respect to $\varepsilon_{j}^{\mathrm{pr}}$ comes from the derivative of the non-linear optimality conditions:

$$
\begin{aligned}
\frac{\partial r_{i}}{\partial \varepsilon_{j}^{\mathrm{pr}}} & =\frac{\partial^{2} \varphi^{\mathrm{e}}}{\partial \varepsilon_{i}^{\mathrm{e}} \partial \varepsilon_{i}^{\mathrm{e}}} \frac{\mathrm{d} \varepsilon_{i}^{\mathrm{e}}}{\mathrm{d} \varepsilon_{j}^{\mathrm{pr}}}-\frac{\partial^{2} \psi}{\partial d_{i}^{\mathrm{v}} \partial d_{i}^{\mathrm{v}}} \frac{\mathrm{d}\left(d_{i}^{\mathrm{v}}\right)}{\mathrm{d} \varepsilon_{j}^{\mathrm{pr}}}+\frac{\mathrm{d} \lambda}{\mathrm{d} \varepsilon_{j}^{\mathrm{pr}}}=0, \quad i, j=1,2,3 \text { (no index sum) } \\
& =\varphi_{, i i}^{\mathrm{e}} \frac{\mathrm{d} \varepsilon_{i}^{\mathrm{e}}}{\mathrm{d} \varepsilon_{j}^{\mathrm{pr}}}-\psi_{, i i}\left(\frac{\delta_{i j}}{\Delta t}-\frac{1}{\Delta t} \frac{\mathrm{d} \varepsilon_{i}^{\mathrm{e}}}{\mathrm{d} \varepsilon_{j}^{\mathrm{pr}}}\right)+\frac{\mathrm{d} \lambda}{\mathrm{d} \varepsilon_{j}^{\mathrm{pr}}}=0 \\
& =\left(\varphi_{, i i}^{\mathrm{e}}+\frac{\psi_{, i i}}{\Delta t}\right) \frac{\mathrm{d} \varepsilon_{i}^{\mathrm{e}}}{\mathrm{d} \varepsilon_{j}^{\mathrm{pr}}}-\left(\psi_{, i i} \frac{\delta_{i j}}{\Delta t}-\frac{\mathrm{d} \lambda}{\mathrm{d} \varepsilon_{j}^{\mathrm{pr}}}\right)=0 \\
& =K_{i i} \frac{\mathrm{d} \varepsilon_{i}^{\mathrm{e}}}{\mathrm{d} \varepsilon_{j}^{\mathrm{pr}}}-\left(\psi_{, i i} \frac{\delta_{i j}}{\Delta t}-\frac{\mathrm{d} \lambda}{\mathrm{d} \varepsilon_{j}^{\mathrm{pr}}}\right)=0 \\
\frac{\partial r_{4}}{\partial \varepsilon_{j}^{\mathrm{pr}}} & =\frac{\mathrm{d} \varepsilon_{1}^{\mathrm{e}}}{\mathrm{d} \varepsilon_{j}^{\mathrm{pr}}}+\frac{\mathrm{d} \varepsilon_{2}^{\mathrm{e}}}{\mathrm{d} \varepsilon_{j}^{\mathrm{pr}}}+\frac{\mathrm{d} \varepsilon_{3}^{\mathrm{e}}}{\mathrm{d} \varepsilon_{j}^{\mathrm{pr}}}=0
\end{aligned}
$$

Isolating $\mathrm{d} \varepsilon_{i}^{\mathrm{e}} / \mathrm{d} \varepsilon_{j}^{\mathrm{pr}}$ from (A9) and substituting in (A10),

$$
\begin{gathered}
\frac{\partial \varepsilon_{i}^{\mathrm{e}}}{\partial \varepsilon_{j}^{\mathrm{pr}}}=\left(\psi_{, i i} \frac{\delta_{i j}}{\Delta t}-\frac{\mathrm{d} \lambda}{\mathrm{d} \varepsilon_{j}^{\mathrm{pr}}}\right) \frac{1}{K_{i i}} \\
\sum_{i=1}^{3} \frac{\psi_{, i i} \delta_{i j}}{\Delta t K_{i i}}-\sum_{i=1}^{3} \frac{\mathrm{d} \lambda}{\mathrm{d} \varepsilon_{j}^{\mathrm{pr}}} \frac{1}{K_{i i}}=0 \\
\frac{\psi_{, j j}}{\Delta t K_{j j}}=\frac{\mathrm{d} \lambda}{\mathrm{d} \varepsilon_{j}^{\mathrm{pr}}} \sum_{i=1}^{3} \frac{1}{K_{i i}}=0 \\
\frac{\mathrm{d} \lambda}{\mathrm{d} \varepsilon_{j}^{\mathrm{pr}}}=\frac{\psi_{, j j}}{\Delta t K_{j j}}\left(\sum_{s=1}^{3} \frac{1}{K_{s s}}\right)^{-1}
\end{gathered}
$$

Then, substituting (A12) in (A11),

$$
\frac{\mathrm{d} \varepsilon_{i}^{\mathrm{e}}}{\mathrm{d} \varepsilon_{j}^{\mathrm{pr}}}=\frac{\psi_{, i i}}{\Delta t K_{i i}} \delta_{i j}-\frac{\psi_{, j j}}{\Delta t K_{j j} K_{i i}}\left(\sum_{s=1}^{3} \frac{1}{K_{s s}}\right)^{-1}
$$




\section{ACKNOWLEDGEMENTS}

Eduardo Fancello would like to thank the Brazilian Coordenação de Aperfeiçoamento de Pessoal de Nivel Superior-CAPES who provided financial support for this research during his stay at LTAS/ULG, Liège, Belgium. He also thanks Ludovic Noels and Luc Papeleux for their valuable help with the code METAFOR.

\section{REFERENCES}

1. Simo JC. On a fully three dimensional finite-strain viscoelastic damage model: formulation and computational aspects. Computer Methods in Applied Mechanics and Engineering 1987; 60:153-173.

2. Holzapfel GA, Simo J. A new viscoelastic constitutive model for continuous media at finite thermomechanical changes. International Journal of Solids and Structures 1996; 33:3019-3034.

3. Holzapfel GA. On large viscoelasticity: continuum formulation and finite element applications to elastomeric structures. International Journal for Numerical Methods in Engineering 1996; 39:3903-3926.

4. Holzapfel GA, Gasser TC. A viscoelastic model for fiber-reinforced composites at finite strains: continuum basis, computational aspects and applications. Computer Methods in Applied Mechanics and Engineering 2001; 190:4379-4403.

5. Bonet J. Large viscoelastic constitutive models. International Journal of Solids and Structures 2001; 38: 2953-2968.

6. Sidoroff F. Un modèle viscoélastique non linèaire avec configuration intermédiaire. Journal de Mécanique 1974; 13:679-713.

7. Lubliner J. A model of rubber viscoelasticity. Mechanics Research Communications 1985; 12:93-99.

8. Kaiss A, Le Tallec P, Rahier C. Three-dimensional incompressible viscoelasticity in large strains: formulation and numerical approximation. Computer Methods in Applied Mechanics and Engineering 1993; 109:223-258.

9. Lion A. A constitutive model for carbon filled rubber experimental investigation and mathematical representations. Continuum Mechanics and Thermodynamics 1996; 8:153-169.

10. Reese S, Govindjee S. A theory for finite viscoelasticity and numerical aspects. International Journal of Solids and Structures 1998; 35:3455-3482.

11. Ortiz M, Stainier L. The variational formulation of viscoplastic constitutive updates. Computer Methods in Applied Mechanics and Engineering 1999; 171:419-444.

12. Radovitzky R, Ortiz M. Error estimation and adaptive meshing in strongly nonlinear dynamic problems. Computer Methods in Applied Mechanics and Engineering 1999; 172:203-240.

13. Stainier L. Une formulation variationelle des algorithmes de calcul des constraintes pour les modèles élastoviscoplastiques et viscoélastiques en grandes transformations. Gème Colloque National en Calcul des Structures, Giens, France, 2003.

14. Simo JC, Taylor RL. Quasi incompressible finite elasticity in principal stretches continuum basis and numerical algorithms. Computer Methods in Applied Mechanics and Engineering 1991; 85:273-310.

15. Mardesen JE, Gurtin ME. Mathematical Foundations of Elasticity. Prentice-Hall: Englewood Cliffs, NJ, EUA, 1983.

16. Holzapfel GA. Nonlinear Solid Mechanics. Wiley: Chichester, England, 2000.

17. de Souza Neto EA, Peric D, Owen DRJ. Computational Methods for Plasticity, in press.

18. Anand L, Weber G. Finite deformations constitutive equations and a time integration procedure for isotropic hyperelastic-viscoplastic solids. Computer Methods in Applied Mechanics and Engineering 1990; 79:173-202.

19. Miehe C. Exponential map algorithm for stress updates in anisotropic multiplicative elastoplasticity for single crystals. International Journal for Numerical Methods in Engineering 1996; 39:3367-3390.

20. Dépt. AéroSpatiale, Mécanique et mAtériaux (ASMA/LTAS-MC\&T), Université de Liège, Belgium. METAFOR-Software for Thermo-mechanical Analysis of Structures in Large Strain Regime. http://garfield. ltas.ulg.ac.be/oo_meta/ 